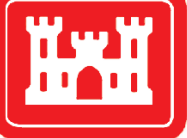

US Army Corps of Engineers ${ }_{\circledast}$

Engineer Research and

Development Center

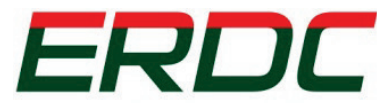

INNOVATIVE SOLUTIONS for a safer, better world

\title{
Early Detection Of Failure Mechanisms In Resilient Biostructures: A Network Flow Study
}

Reena R. Patel, Guillermo A. Riveros, David S. Thompson,

October 2017

Felipe J. Acosta, Edward J. Perkins, Jan J. Hoover,

John F. Peters, and Antoinette Tordesillas

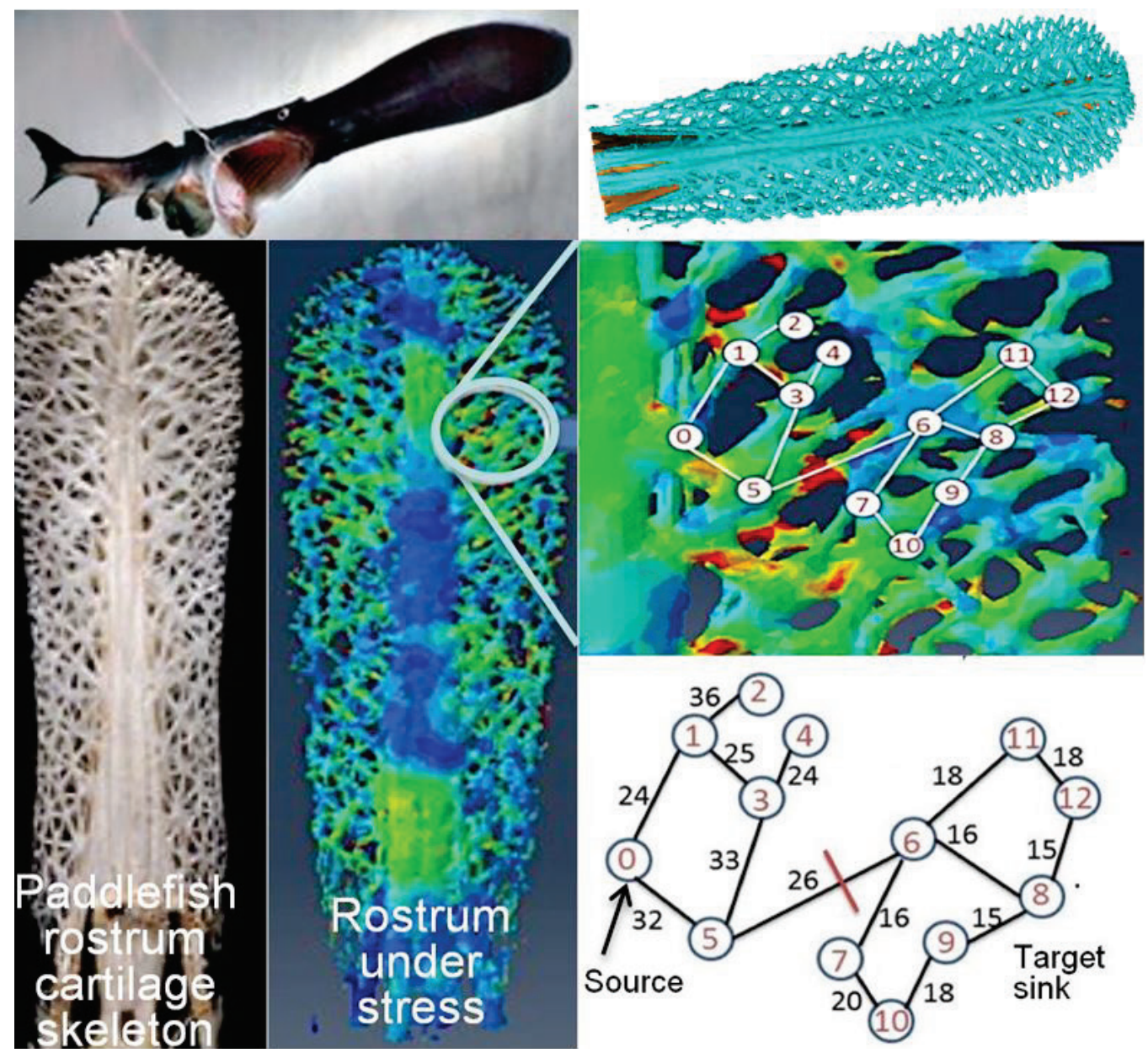


The US Army Engineer Research and Development Center (ERDC) solves the nation's toughest engineering and environmental challenges. ERDC develops innovative solutions in civil and military engineering, geospatial sciences, water resources, and environmental sciences for the Army, the Department of Defense, civilian agencies, and our nation's public good. Find out more at www.erdc.usace.army.mil.

To search for other technical reports published by ERDC, visit the ERDC online library at http://acwc.sdp.sirsi.net/client/default. 


\section{Early Detection Of Failure Mechanisms In Resilient Biostructures: A Network Flow Study}

Reena R. Patel and Guillermo A. Riveros

Information Technology Laboratory

U.S. Army Engineer Research and Development Center 3909 Halls Ferry Rd.

Vicksburg, MS 39180-6199

Edward J. Perkins and Jan J. Hoover

Environment Laboratory

U.S. Army Engineer Research and Development Center 3909 Halls Ferry Rd.

Vicksburg, MS 39180-6199

Felipe J. Acosta

University of Puerto Rico-Mayaguez

University of Puerto Rico, Mayaguez Campus

Mayaguez, PR 00681-9000

David S. Thompson and John F. Peters

Mississippi State University

B.S. Hood Rd.

Starkville, MS 39762

Antoinette Tordesillas

The University of Melbourne

Victoria 3010

Final report

Approved for public release; distribution is unlimited.

Prepared for U.S. Army Corps of Engineers

Washington, DC 20314-1000

Under Project WP \# 17-202, “Complex Network Analysis for Early Detection of Failure Mechanisms in Resilient Biostructures" 


\section{Abstract}

The area of bioinspired material design is fairly young and mainly unexploited. This research aims to gain fundamental understanding of the hierarchical lattice architecture in the paddlefish rostrum and use this knowledge to obtain new insights for a wide range of applications. This work presents an integrated, interdisciplinary approach that employs computational mechanics and the theory of network statistics to gain fundamental insights into the failure mechanisms of high performance, lightweight, structured composites by examining the geometry and material properties of the rostrum. Results from computational mechanics simulations and network flow analysis are presented with emphasis on the load transfer mechanism in the Bio-Structure. The structure is formed from a network of structural elements that carries forces through combinations, or chains, of structural members called force chains in reference to similar structures generally observed in granular media. These force chains are often aligned in the principal direction of the external stress to which the system is subjected. The current research presents a flow network analysis of the rostrum with and without the presence of the lattice architecture. Highly localized force chains were formed in the rostrum with similar loading conditions in absence of the lattice architecture. The flow network was also able to capture the stain localization in the tensile region of the rostrum and the asymmetrical response of the rostrum to uniform loading.

DISCLAIMER: The contents of this report are not to be used for advertising, publication, or promotional purposes. Citation of trade names does not constitute an official endorsement or approval of the use of such commercial products. All product names and trademarks cited are the property of their respective owners. The findings of this report are not to be construed as an official Department of the Army position unless so designated by other authorized documents. 


\section{Contents}

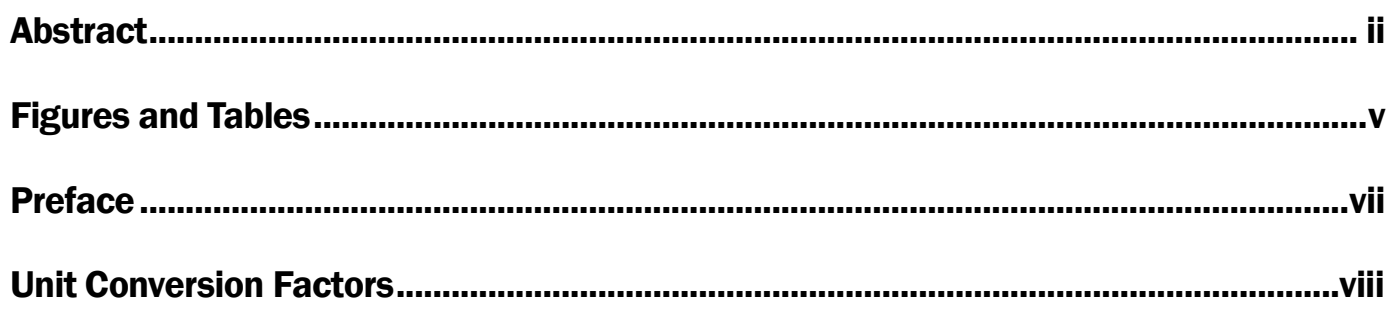

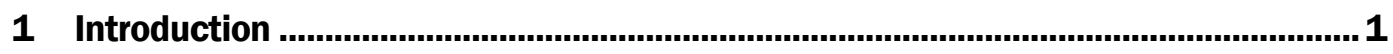

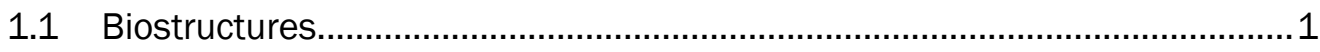

1.2 Previous research on rostrum.................................................................. 2

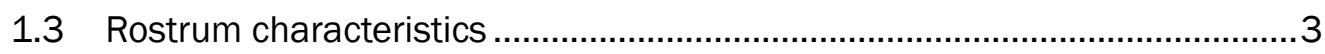

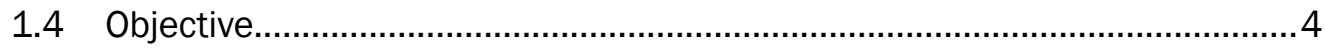

1.5 Novel transdisciplinary approach ...................................................... 5

2 Computational Mechanics Experiments on the Rostrum.......................................... 7

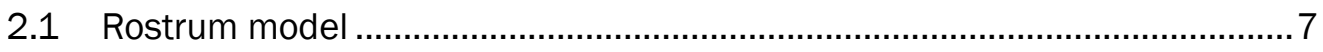

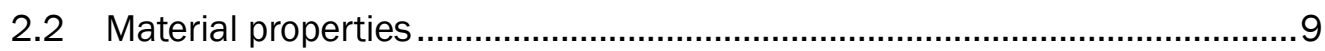

2.3 Force and displacement boundary conditions ...........................................10

2.4 Computational solid mechanics analysis..............................................11

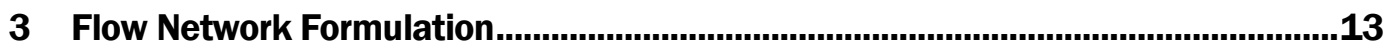

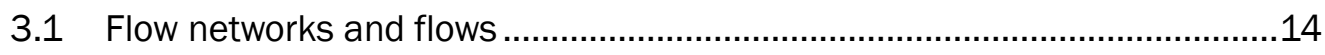

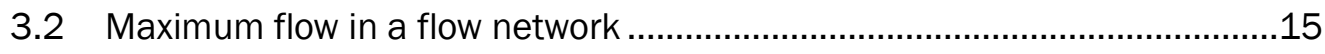

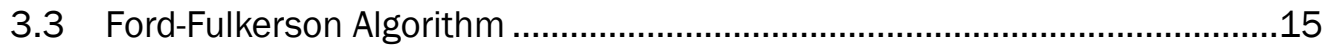

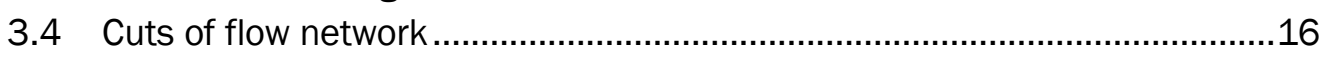

3.5 Maximum flow minimum cut theorem ........................................................16

3.6 Formulate the rostrum as a network flow problem ....................................16

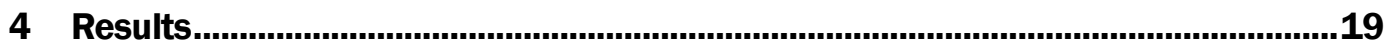

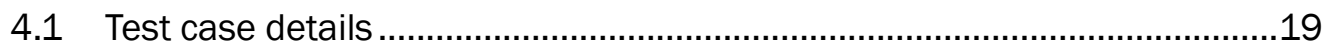

4.2 Stress distribution in the rostrum subjected to uniform pressure ............... 20

4.3 Stress distribution in the rostrum subjected to uniform pressure: tissue.

4.4 Stress distribution in the rostrum subjected to a uniform pressure:

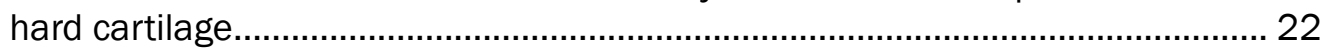

4.5 Stress distribution in the rostrum subjected to a uniform pressure:

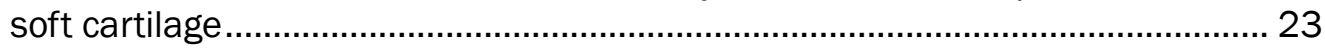

4.6 Network flow analysis of the load distribution with increasing

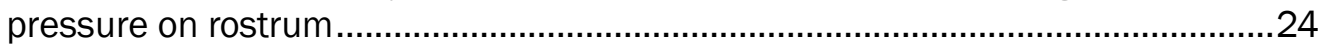

4.7 Stress distribution in the rostrum without the lattice architecture ..............27

4.8 Network flow analysis of the load distribution with increasing pressure on the rostrum without the lattice architecture .....................................27

4.9 Analytical verification of methodology....................................................... 28 


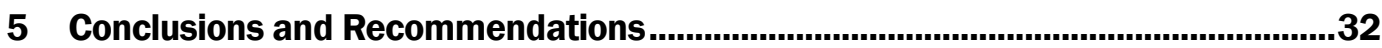

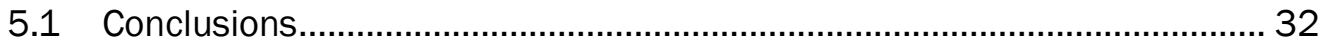

5.2 Load transfer mechanisms for the rostrum ................................................. 32

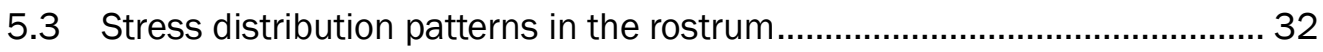

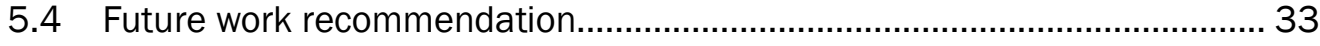

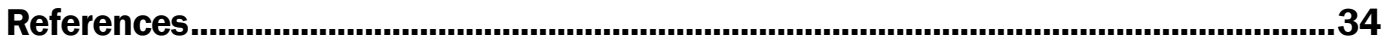

Report Documentation Page 


\section{Figures and Tables}

\section{Figures}

Figure 1. Paddlefish (Photo: Wikimedia Commons).

Figure 2. Paddlefish rostrum cartilage skeleton (http://www.Imrcc.org/paddlefish-

primitive-fish-inspiring-future-technology/).

Figure 3. Tissue.

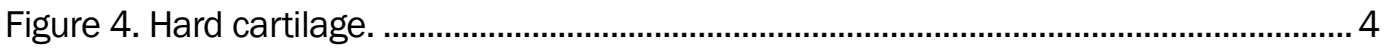

Figure 5. Soft cartilage............................................................................................... 4

Figure 6. Cross-section (A-A, displayed in Figure 3) of rostrum displaying three parts shown in Figure 3 through Figure 5 ..................................................................... 4

Figure 7. Interdisciplinary approach flowchart. .................................................................. 6

Figure 8. X-Ray CT scans performed on rostrum......................................................... 8

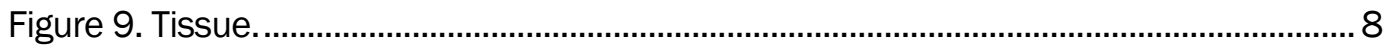

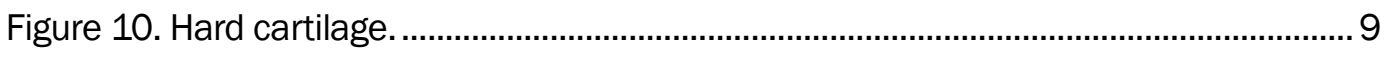

Figure 11. Soft cartilage. ............................................................................................... 9

Figure 12. Assembly of the three components of rostrum (i.e., tissue, hard

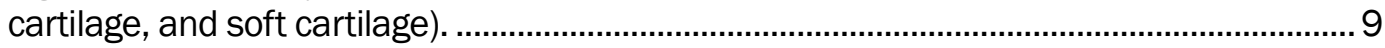

Figure 13. Stress-strain curve for steel......................................................................... 10

Figure 14. Uniform pressure loading on rostrum with fixed support..................................11

Figure 15. Garnet. Cray XE6 - 1.5 PFLOPS (Courtesy of ERDC-HPC-DSRC).........................12

Figure 16. A demonstration model constructed from a small part of the rostrum to illustrate the modus operandi of maximum flow-minimum cut (MFMC) algorithm.

Figure 17. Contour plot of Von-Mises Stresses for the three components of rostrum. Yielding regions are circled for clarity.

Figure 18. Stress distribution due to uniform pressure loading on rostrum: tissue...........22

Figure 19. Stress distribution due to uniform pressure loading on rostrum: hard cartilage.

Figure 20. Stress distribution due to uniform pressure loading on rostrum: soft cartilage.

Figure 21. Network flow analysis of load distribution with increasing pressure on rostrum's bottom surface.

Figure 22. Network flow analysis of load distribution with increasing pressure on rostrum's top surface.

Figure 23. Rostrum without lattice architecture.

Figure 24. Network flow analysis of load distribution with increasing pressure on rostrum without lattice architecture.

Figure 25. Simply supported beam with concentrated load at the center. ........................30

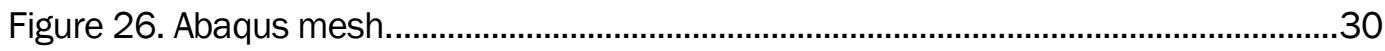

Figure 27. Von-Mises stresses...................................................................................... 31 


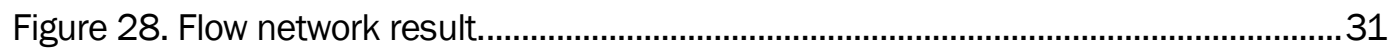
Tables

Table 1. Details of the computational mesh of the rostrum.............................................. 8 


\section{Preface}

This study was conducted for the Headquarters U.S. Army Corps of Engineers (USACE) Program under Project WP \# 17-202, "Complex Network Analysis for Early Detection of Failure Mechanisms in Resilient Biostructures." This work was a direct result of technology transfer from Dr. Tordesillas's visit to ERDC through award W911NF-15-1-0292. The technical monitor was Dr. Edward J. Perkins.

The work was performed by the Computational Analysis Branch (CAB) of the Computational Science and Engineering Division (CSED), U.S. Army Engineer Research and Development Center, Information Technology Laboratory (ERDC-ITL). At the time of publication, Dr. Jeffery L. Hensley was Chief, CEERD-IE-C; Dr. Jerrell R. Ballard was Chief, CEERD-IE; and Mr. David R. Richards, CEERD-IV-T was the Technical Director for Engineering and Science. The Deputy Director of ERDC-ITL was Ms. Patti S. Duett and the Director was Dr. Reed L. Mosher.

The authors acknowledge the financial support provided by the U.S Army Engineer Research and Development Center (ERDC) Military Engineering 6.1 Basic Research program. Contributions made by Mississippi State University are greatly appreciated.

COL Bryan S. Green was the Commander of ERDC, and Dr. David W. Pittman was the Director. 


\section{Unit Conversion Factors}

\begin{tabular}{|l|c|l|}
\hline Multiply & By & To Obtain \\
\hline cubic inches & $1.6387064 \mathrm{E}-05$ & cubic meters \\
\hline inches & 0.0254 & meters \\
\hline inch-pounds (force) & 0.1129848 & newton meters \\
\hline pounds (force) & 4.448222 & newtons \\
\hline pounds (force) per foot & 14.59390 & newtons per meter \\
\hline pounds (force) per inch & 175.1268 & newtons per meter \\
\hline pounds (force) per square inch & 6.894757 & kilopascals \\
\hline pounds (mass) & 0.45359237 & kilograms \\
\hline pounds (mass) per cubic foot & 16.01846 & kilograms per cubic meter \\
\hline pounds (mass) per cubic inch & 2.757990 & kilograms per cubic meter \\
\hline pounds (mass) per square foot & E+04 & \\
\hline pounds (mass) per square yard & 4.882428 & kilograms per square meter \\
\hline slugs & 0.542492 & kilograms per square meter \\
\hline square inches & 14.59390 & kilograms \\
\hline
\end{tabular}




\section{Introduction}

\subsection{Biostructures}

Nature has optimized complex Biostructures over billions of years to have remarkable resiliency, strength, and lightweight composition. Biological systems such as bone and oyster shells are strong and energy dissipative because of the arrangement of organic and inorganic materials (Bruce and O'Hare 1996). The Engineer Research Development Center, Information Technology Laboratory (ERDC-ITL) proposes to gain fundamental insights into the design and engineering of high performance, lightweight, structured composites by examining the geometry and material properties of the paddlefish rostrum (Figure 1 and Figure 2).

The rostrum contains a skeletal lattice structure that is complex, yet light weight with unique properties. The lattice structure itself has a geometry that is non-uniform. An earlier feasibility study conducted on the rostrum led to the conclusion that the non-uniform geometry is one of nature's toughening mechanisms used to mitigate failure* (Patel and Riveros 2013; Allen and Riveros 2013). A fundamental understanding of how this latticelike architecture functions has the potential to provide new insights into applications over a wide range of disciplines (e.g., protective panels, novel building materials, body and vehicle armor, and ship design among other possible uses).

\footnotetext{
* Riveros, G. A., R. R. Patel, and J. J. Hoover. In prep. Swimming enhancement induced by the rostrum of the paddlefish (Polyodon spathula) in laminar flows: A multiphysics, fluid-structure interaction analysis. Submitted to Applied Mathematical Modelling.
} 
Figure 1. Paddlefish (Photo: Wikimedia Commons).

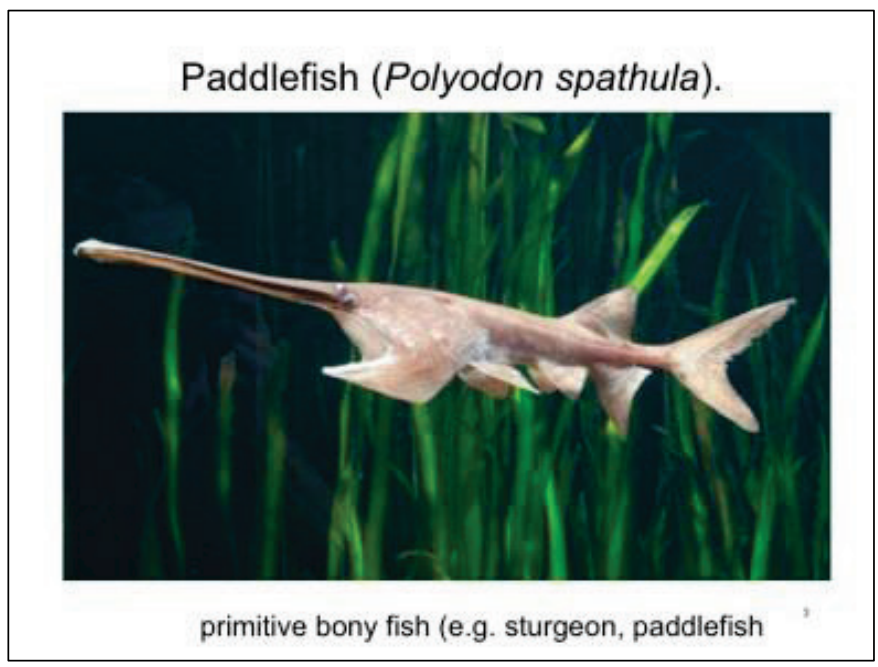

Figure 2. Paddlefish rostrum cartilage skeleton (http://www.Imrcc.org/paddlefish-primitive-fish-inspiring-future-technology/).

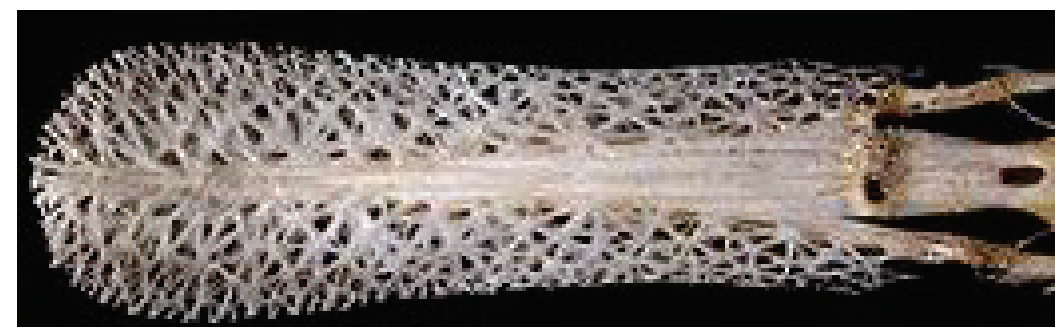

\subsection{Previous research on rostrum}

Previous research conducted on the paddlefish rostrum revealed the following: (a) the rostrum of the North American Paddlefish is distinct and complex with no functional counterpart in any other species. It is long, broad, and flat, rounded at the tip, and rhomboidal in cross-section. The cartilage endoskeleton consists of a central gelatinous filled tube, flanked by two buttresses and supports a lattice-like network of small inter-connecting stellate bones (Allen and Riveros 2013; Allison et al. 2013; Allis 1903; Davis 1959). The lattice and bone diameters are larger near the center of the rostrum and smaller near the edge; however, the pattern is not obvious. The relative size and shape of the rostrum are highly variable in juveniles (Grande and Bemis 1991), but less so in sub-adults and adults (Hoover et al. 2009). Rostra in other species of fish consist of flat blades of solid cartilage (sawfishes and some sharks) or simple tubes of bone (swordfish, marlin, etc.) and do not vary appreciably in size and shape within species ( $\mathrm{J} \mathrm{J}$. Hoover 2000). (b) Swimming enhancement induced by the rostrum has 
been extensively studied* (Patel and Riveros 2013; Allen and Riveros 2013). Recent computational analysis revealed the rostrum has far greater attributes of energy dissipation and impact resistance when compared to man-made engineered systems (Riveros et al. 2015). (C) The authors hypothesize that the rostrum's lattice structure is the major contributor to this superior performance. (D) However, the lattice is considered a statically indeterminate, non-linear structure with varying material types, and material properties, with non-uniform stiffness and irregularly shaped members. Knowledge on how the structure is geometrically assembled to possess high-performance strength/toughness characteristics is lacking.

\subsection{Rostrum characteristics}

The paddlefish rostrum displays unique strength that evolved from the ancient cartilaginous fish species, yet it is unclear what cellular and biochemical features interact during the fish's development to give the rostrum its unique strength/resilience. The complexities of this biological system and the lack of knowledge about biomaterials have introduced complications for experimental approaches that seek to understand the biomechanical geometrical behaviors of soft tissues. Therefore, it is necessary to understand the role redundant hierarchical lattice members have in achieving the observed structures resiliency.

Biological structures have a complex interplay of not only complex geometry but also heterogeneous material properties. This complex interplay produces uncertainty as to what is dictating the structural response. Figure 3 displays the outer soft tissue layer of the rostrum. Figure 4 shows the inner hard cartilage of the rostrum, which has an elastic modulus of an order of magnitude stiffer than the tissue. Figure 5 is representative of the inner soft cartilage of the rostrum whose elastic modulus is an order of magnitude softer than the hard cartilage. Figure 6 shows the cross section of the rostrum displaying its three components. As displayed in Figure 3 through Figure 6, there is interplay of geometry and material properties. It is hypothesized that this complexity in lattice geometry and material properties makes the rostrum lightweight yet energy dissipative.

\footnotetext{
${ }^{*}$ Riveros, G. A., R. R. Patel, and J. J. Hoover. In prep. Swimming enhancement induced by the rostrum of the paddlefish (Polyodon spathula) in laminar flows: A multiphysics, fluid-structure interaction analysis. Submitted to Applied Mathematical Modelling.
} 
Figure 3. Tissue.

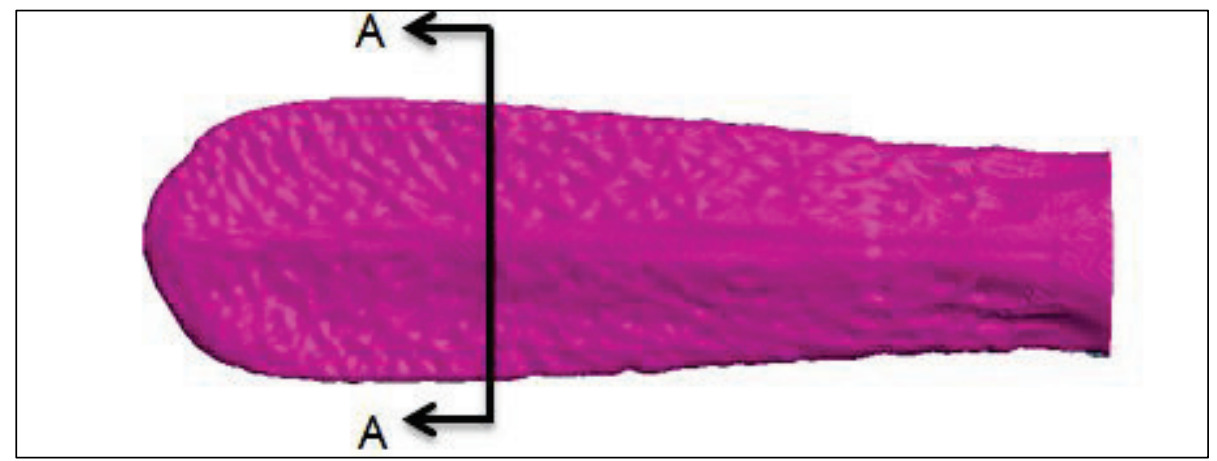

Figure 4. Hard cartilage.

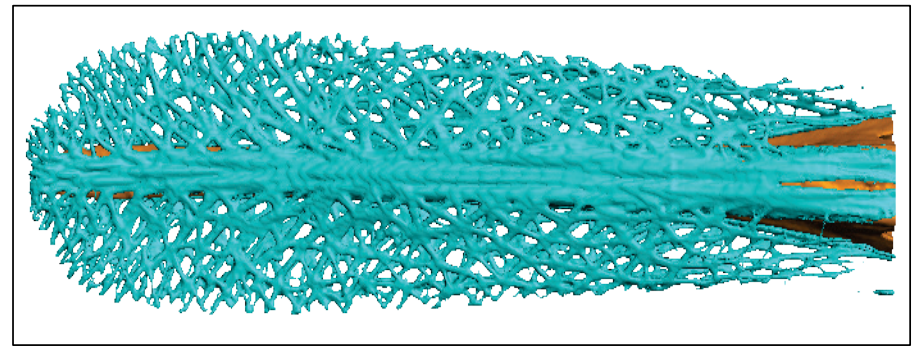

Figure 5. Soft cartilage.

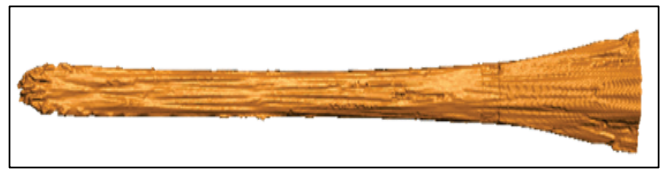

Figure 6. Cross-section (A-A, displayed in Figure 3 ) of rostrum displaying three parts shown in Figure 3 through Figure 5.

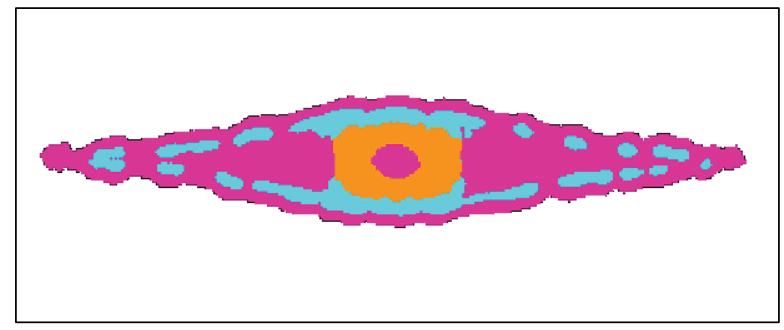

\subsection{Objective}

To obtain fundamental understanding of failure mechanisms in resilient Biostructures, it is essential to gain insight into the underlying kinematics of the system that play a vital role in load transfer and formation of the stress flow patterns in the network. The objectives of the current research work are as follows: 
1. Develop three-dimensional (3D) models of the rostrum from computerized tomography (CT) scans.

2. Conduct computational mechanics experiments on the rostrum with and without the redundant lattice architecture.

3. Formulate the rostrum as a network flow problem from the nodes and connectivity information obtained from the computational mechanics experiments.

4. Show the formation of the stress distribution pattern when the rostrum is subjected to an external loading condition.

5. Verify the approach with analytical modeling.

\subsection{Novel transdisciplinary approach}

This research effort will make use of unique embryonic technologies to investigate the emergent failure resistant mechanisms of the rostrum. For the first time, knowledge will be gained that better describes how nature optimized the skeletal structure, which appears to exhibit mechanical strength/resilience as well as efficient hydrodynamic attributes. Figure 7 provides a flowchart of the ongoing research effort. As depicted in Figure 7, the first step will involve constructing a 3D model using multiple CT scans of the rostrum. Numerical representations will be developed from the $3 \mathrm{D}$ model. The current research will involve conducting computational mechanics experiments (simulations) on the rostrum as it is subjected to a uniform pressure loading condition on the upper surface of the rostrum. Although simulations give an overall distribution of stresses in the system, the underlying details of the changing load transfer mechanisms of the systems is still missing. These mechanisms play a vital role in formation of stress distribution pattern governed by major load bearing members of the structural systems. Towards this end, the loading of the rostrum will be formulated as a network flow problem. The nodes and edges of the rostrum's network will be extracted from the model used in the computational mechanics experiments from the nodes and connectivity information. The flow network will be weighted based on the parameter of interest, which may be stress, energy dissipation etc. The weights of the network will be extracted from the output database file generated from the computational mechanics experiment on the biostructure. The kinematics of the structural system changes when it is under the influence of an external loading condition. The changing kinematics of the system is input to the mathematical algorithm that will compute the maximum flow of the stresses at uniform cost. In structural system, this problem would be formulated as finding the maximum amount of stress to flow through the 
system with minimum disruption to the structural system. Mathematical techniques are required to understand how the stress distribution patterns are formed. This report discusses the load transfer mechanisms for the rostrum of the paddlefish by conducting computational mechanics experiments and identifying the formation of the stress distribution pattern in the rostrum by employing maximum flow/minimum cut mathematical algorithm.

Figure 7. Interdisciplinary approach flowchart.

Complex Network Analysis for Early Detection of Failure Mechanisms in Resilient BioStructures

Hypothesis: The rostrum has a complex lattice(fractal-like) skeletal structure that appears to contribute to enhanced toughness mechanisms, if this is true, we predict that simplifying the lattice structure will result in less resilient structures.

Innovative approach

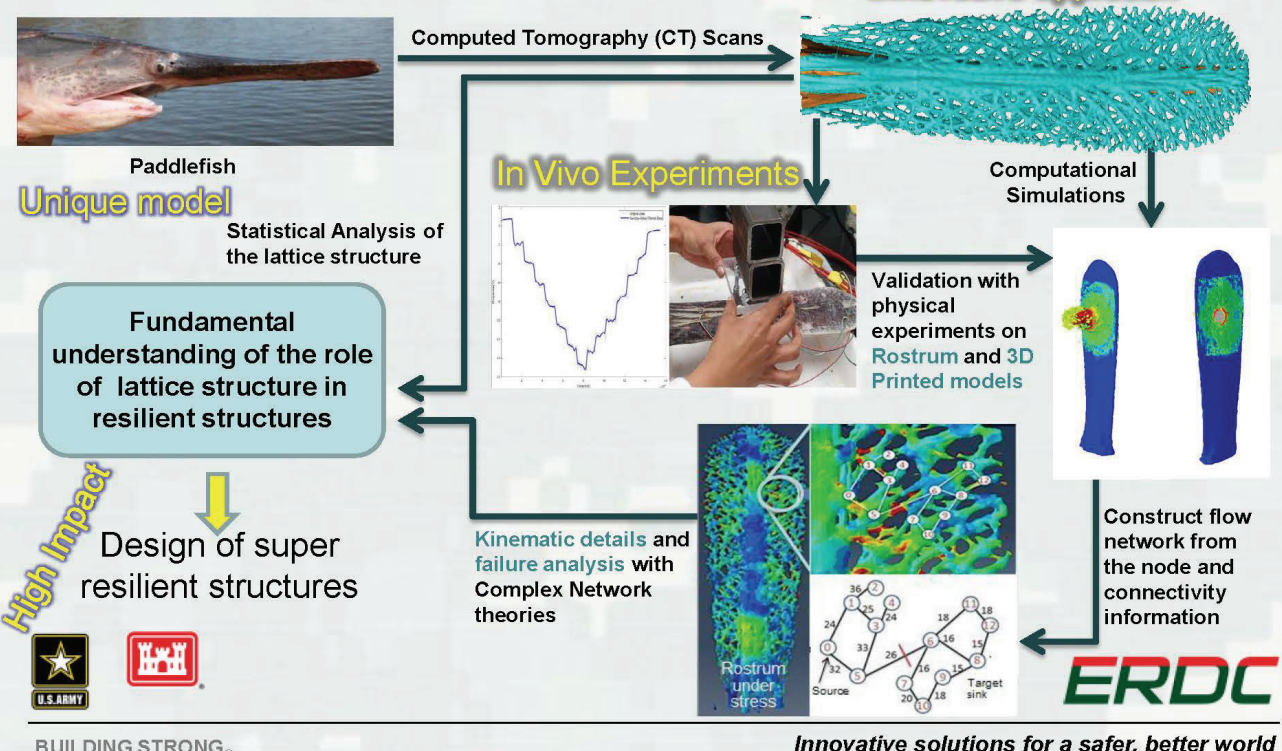




\section{Computational Mechanics Experiments on the Rostrum}

Computational mechanics has been successfully used to simulate complex engineering phenomenon for materials and structures by coupling modelling techniques with high performance computing implementations. Advanced computational structural mechanics simulations with over 3 million degrees of freedom (DOF) provide an important tool for analyzing the structural resiliency and are an irreplaceable part of many industrial and academic areas of study. High fidelity analyses of structures are supremely important, not only for designing new structures but also for ongoing assessment of critical infrastructure. To obtain accurate solutions, it is necessary to construct models with high-order elements and millions of DOF; furthermore, explicit formulations are typically required to obtain useful solutions. Biostructures have been optimized by nature to possess remarkable resiliency and strength with a light-weight composition. The hierarchical geometrical arrangements that are prevalent in Biostructures, coupled with heterogeneous constituents, presents uncertainty as to what dictates the material response. Towards this end, high performance computational mechanics simulations are carried out on a challenging BioStructural domain. The complexity and volume of data involved in such an analysis requires the use of high performance computing environment.

\subsection{Rostrum model}

An X-ray CT was performed on the paddlefish rostrum using the Phoenix $\mathrm{v} \mid$ tome|x. A $3 \mathrm{D}$ model of the rostrum was created by combining multiple CT scans (Deang et al. 2016). Figure 8 shows the rostrum of the paddlefish and the X-ray CT scan that was developed by combining multiple CT scans. The mesh for the computational model was obtained from the tomography (CT scan) of the paddlefish rostrum. Figure 9 shows outermost layer of tissue of the rostrum; Figure 10 shows the hard cartilage; Figure 11 shows the innermost soft cartilage. The soft cartilage is located in the central portion of the rostrum. The hard cartilage branches out from the center of the rostrum toward the width of the rostrum as represented in Figure 8 and Figure 10. The computational models of the three parts are individually imported into the commercial software Abaqus (Figure 12). These complex parts are meshed using a combination of hexahedral and tetrahedral elements. The details of the mesh are provided in Table 1. 
Table 1. Details of the computational mesh of the rostrum.

\begin{tabular}{|c|c|c|c|c|}
\hline Rostrum Part & Element Type & Element Shape & $\begin{array}{c}\text { Geometric } \\
\text { Order }\end{array}$ & Elements \\
\hline \multirow[t]{4}{*}{ Hard Cartilage } & C3D8 & Hexahedral & Linear & 10943 \\
\hline & C4D4 & Tetrahedral & Linear & 303203 \\
\hline & Total nodes & \multicolumn{3}{|c|}{105747} \\
\hline & Total elements & \multicolumn{3}{|c|}{314146} \\
\hline \multirow[t]{4}{*}{ Soft Cartilage } & C3D8 & Hexahedral & Linear & 15745 \\
\hline & C4D4 & Tetrahedral & Linear & 146476 \\
\hline & Total nodes & \multicolumn{3}{|c|}{53991} \\
\hline & Total elements & \multicolumn{3}{|c|}{162221} \\
\hline \multirow[t]{4}{*}{ Tissue } & C3D8 & Hexahedral & Linear & 93024 \\
\hline & C4D4 & Tetrahedral & Linear & 850772 \\
\hline & Total nodes & \multicolumn{3}{|c|}{303263} \\
\hline & Total elements & \multicolumn{3}{|c|}{943796} \\
\hline
\end{tabular}

Figure 8. X-Ray CT scans performed on rostrum.

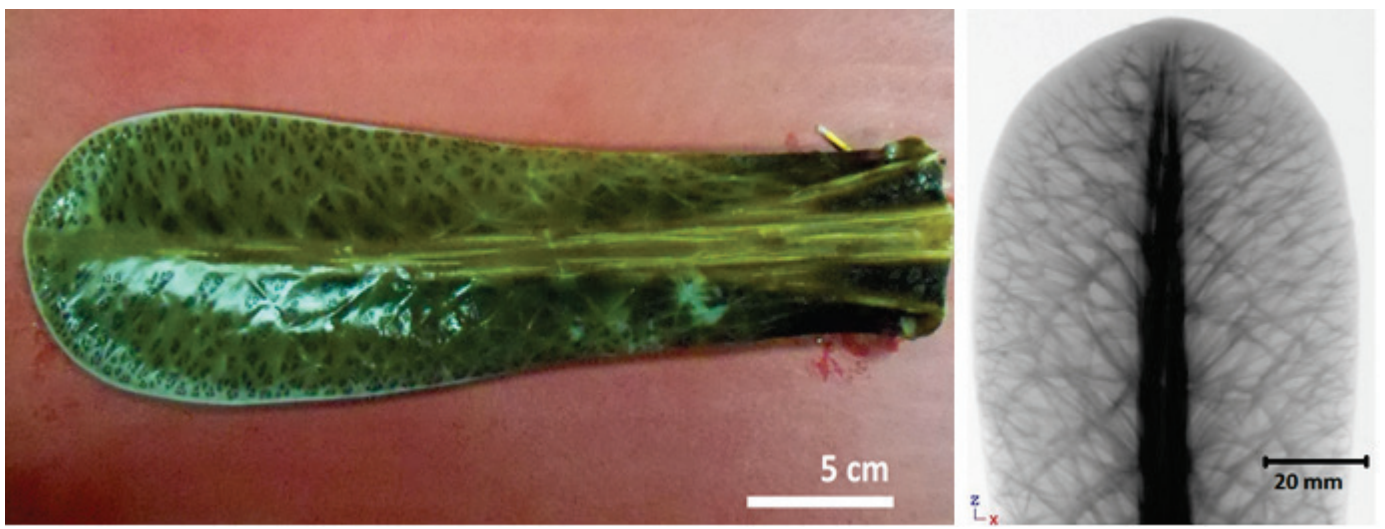

Figure 9. Tissue.

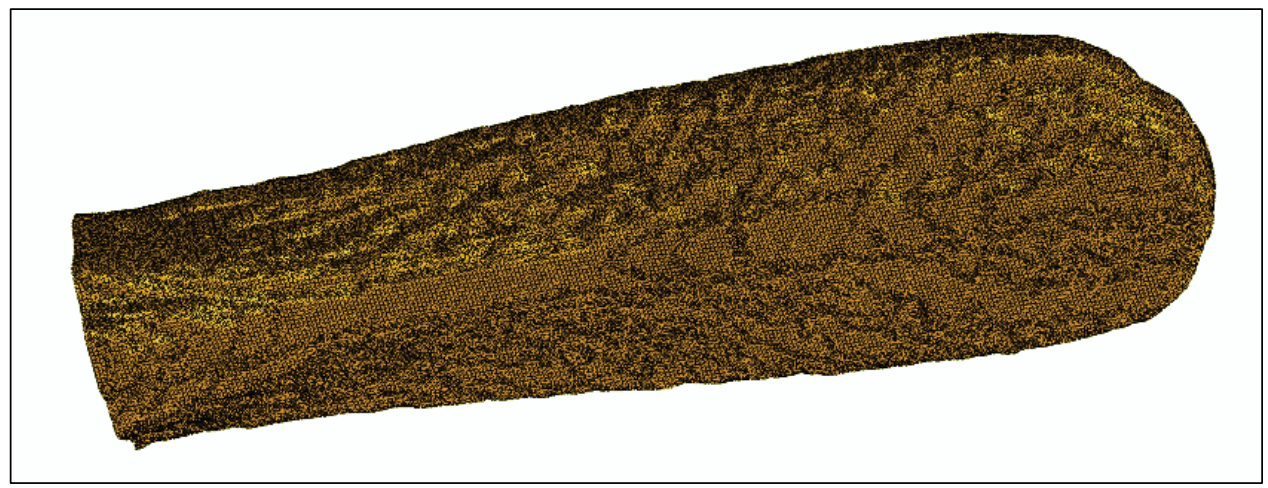


Figure 10. Hard cartilage.

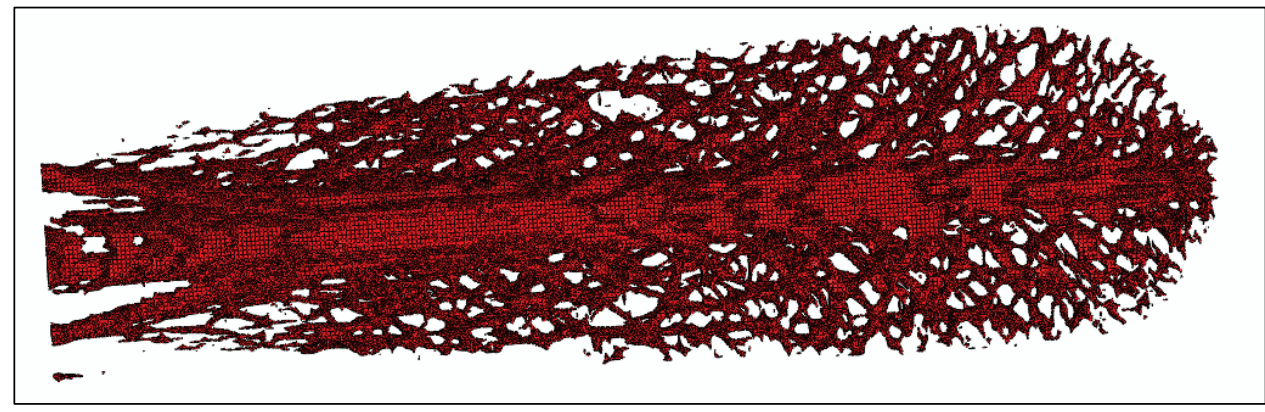

Figure 11. Soft cartilage.

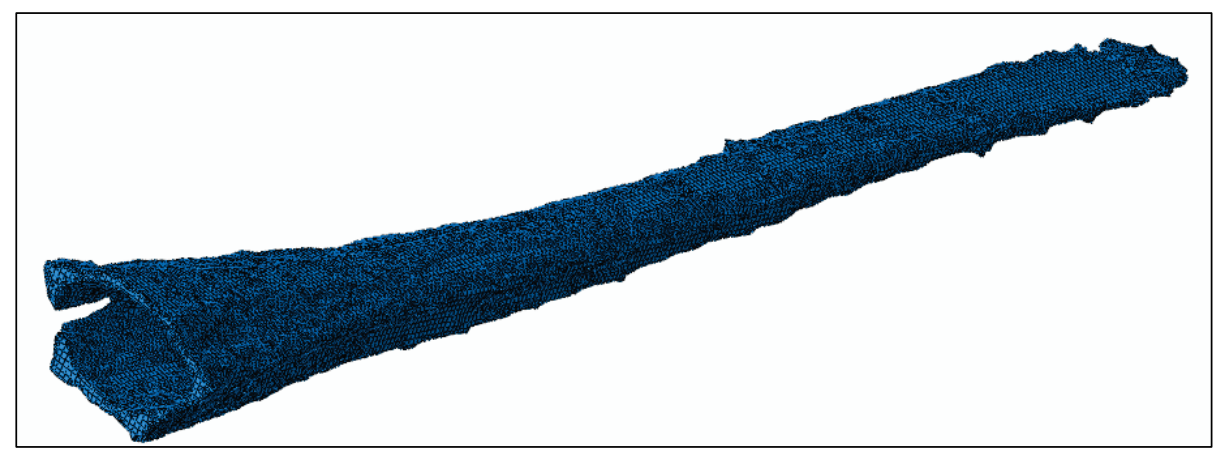

Figure 12. Assembly of the three components of rostrum (i.e., tissue, hard cartilage, and soft cartilage).

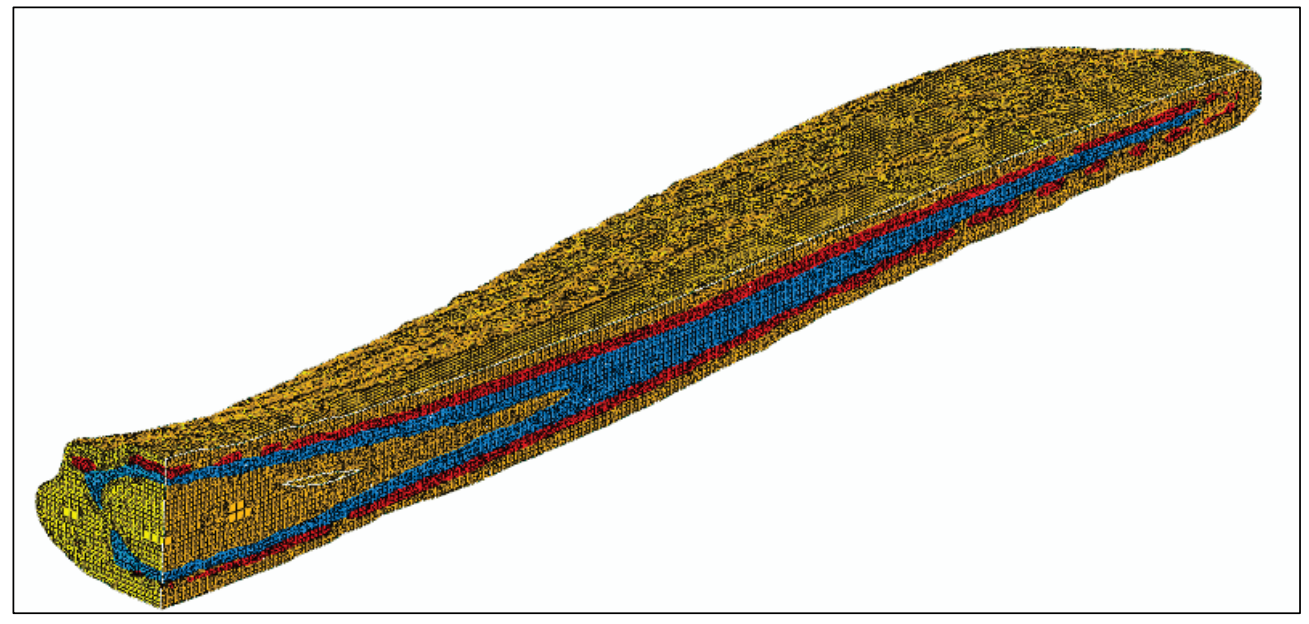

\subsection{Material properties}

Steel with elastic plastic behavior is used in the computational experiments to represent the tissue, hard cartilage, and soft cartilage components of the rostrum in a simple model to demonstrate the progressive yielding of the rostrum. This simple material model is used to focus attention on the geometry of the rostrum and the mathematical model to illustrate the formation of the force chains and identify the load 
transfer mechanisms in the Bio-Structure. To demonstrate the response of the rostrum to a uniform pressure loading, steel, with an elastic modulus of 200,000 MPa, Poisson's ratio of 0.3, yield stress of $345 \mathrm{MPa}$, yield strain of $0.00172 \mathrm{~mm} / \mathrm{mm}$, and mass density $7,850 \mathrm{~kg} / \mathrm{m}^{3}$, is used for the computational mechanics experiments. The value of failure strain used is $0.002 \mathrm{~mm} / \mathrm{mm}$, and damage energy function is linear with zero energy for abrupt failure. Figure 13 displays the material properties of the elasticplastic material (steel) used in the current study. The ultimate stress is 545 $\mathrm{MPa}$. The Abaqus model uses a plastic curve represented by the true stress and plastic strain. The model assumes a perfectly plastic behavior beyond the ultimate stress (545 MPa). In the elastic regime, Abaqus uses an isotropic elastic model parameterized by Young's modulus and Poisson's ratio. Beyond the yield point, and up to the ultimate stress, the response is controlled by strain hardening plasticity.

Figure 13. Stress-strain curve for steel.

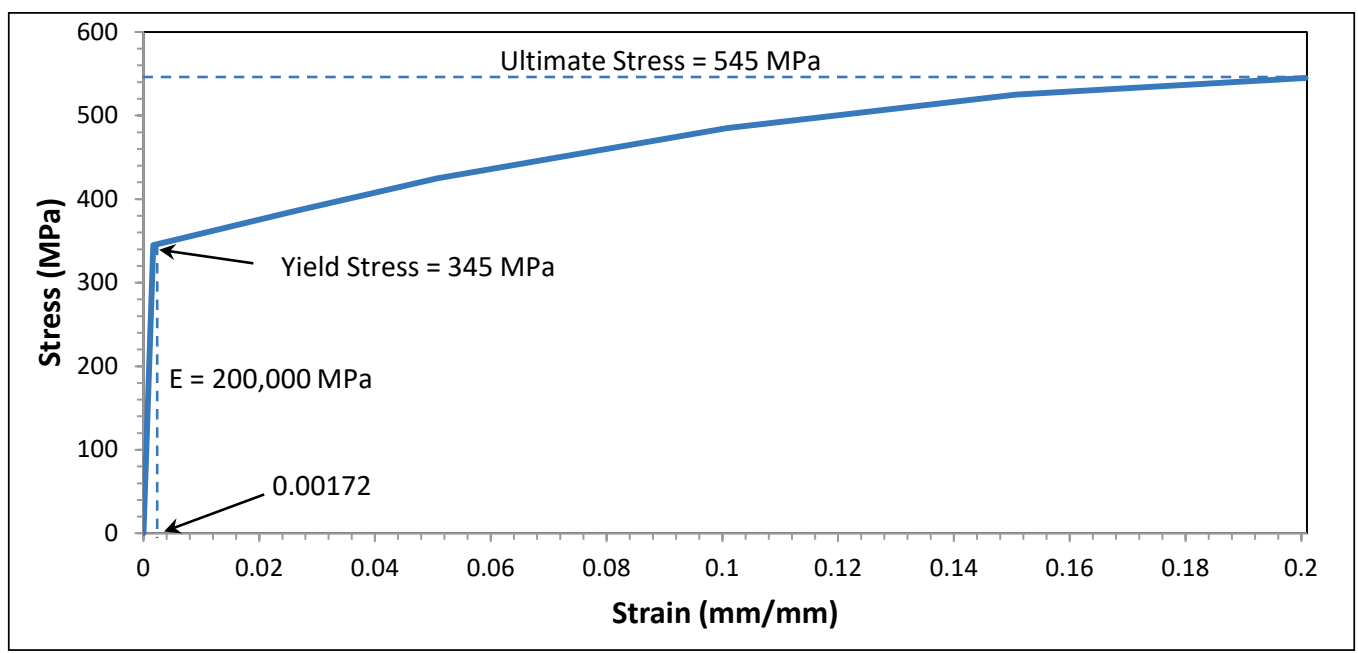

\subsection{Force and displacement boundary conditions}

Figure 14 displays the loading direction of the uniform pressure applied to the upper surface of the rostrum (represented by the purple shading). Gravity is applied to the entire model in the negative Y-direction. The rostrum is modeled similar to a fixed, supported plate by restraining the $(\mathrm{X}, \mathrm{Y}, \mathrm{Z})$ components of displacement. The total weight of the model is $18.58 \mathrm{~N}$, which is comparatively smaller than the applied uniform pressure force. Since the elements used do not consider rotational degrees of freedom, it is sufficient to restrain displacements in three principal directions. A uniform pressure of $50 \mathrm{MPa}$ is applied along the loading direction as specified in Figure 14. 
Figure 14. Uniform pressure loading on rostrum with fixed support*.

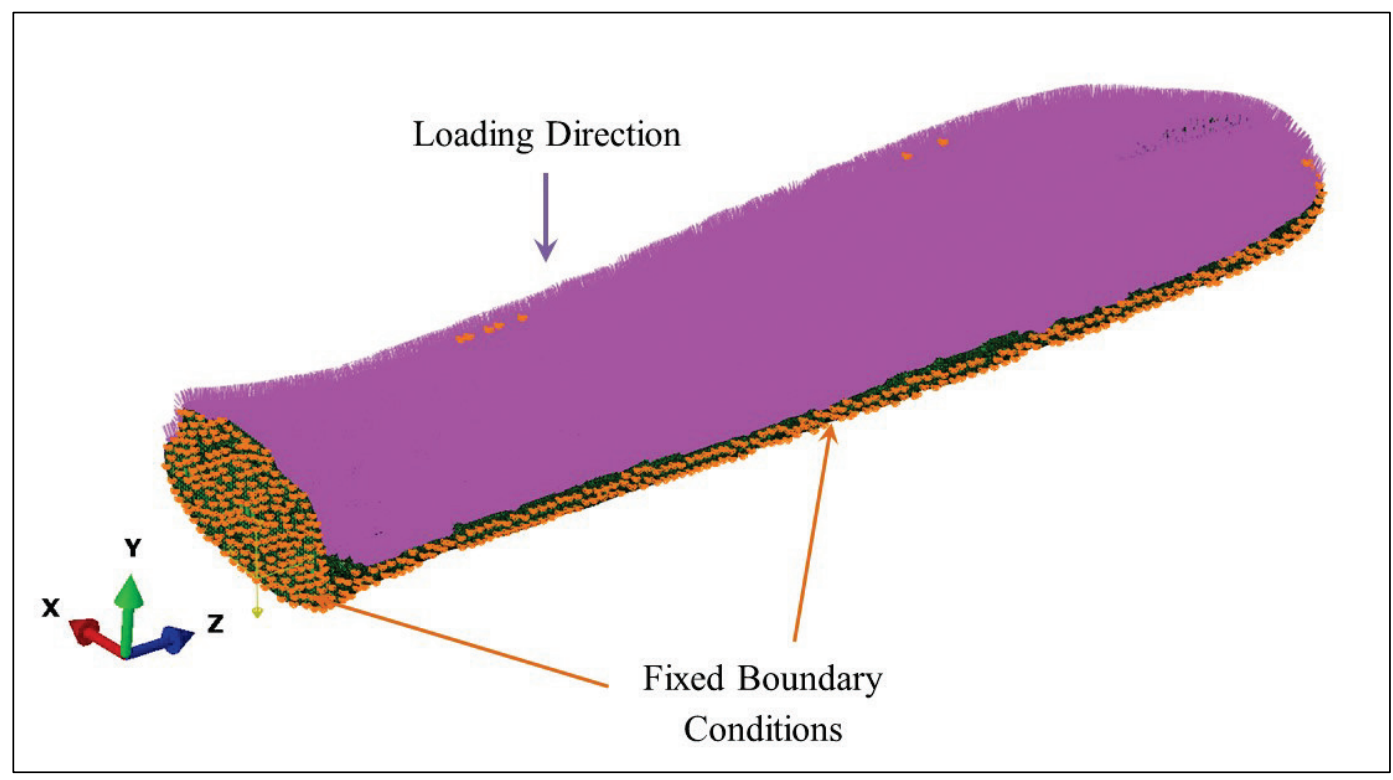

\subsection{Computational solid mechanics analysis}

The simulations are carried out using Abaqus 6.13. A general static analysis is conducted on the rostrum through Abaqus/standard. Gravity is applied in the initial step of the analysis. A uniform surface pressure is applied after the initialization step using a set of increments starting with 0.1 and ramping all the way to the maximum magnitude. The nonlinearity of the geometry was taken into account in the analysis. Owing to the complexity of the geometry and the non-linear analysis, each job was executed on ERDC's High Performance Computing facilities located in Vicksburg, Mississippi. Figure 15 shows the image of the Cray XE6 - 1.5 PFLOPS (Garnet) located at the ERDC's High Performance Computing Modernization Program (HPCMP) Department of Defense (DoD) Supercomputing Resource Center (DSRC).

\footnotetext{
* Acosta, F. J., G. A. Riveros, R. R. Patel, and W. Hodo. In prep. Numerical simulation of biological structures: Paddlefish Rostrum. ERDC Technical report.
} 
Figure 15. Garnet. Cray XE6 - 1.5 PFLOPS (Courtesy of ERDC-HPC-DSRC).

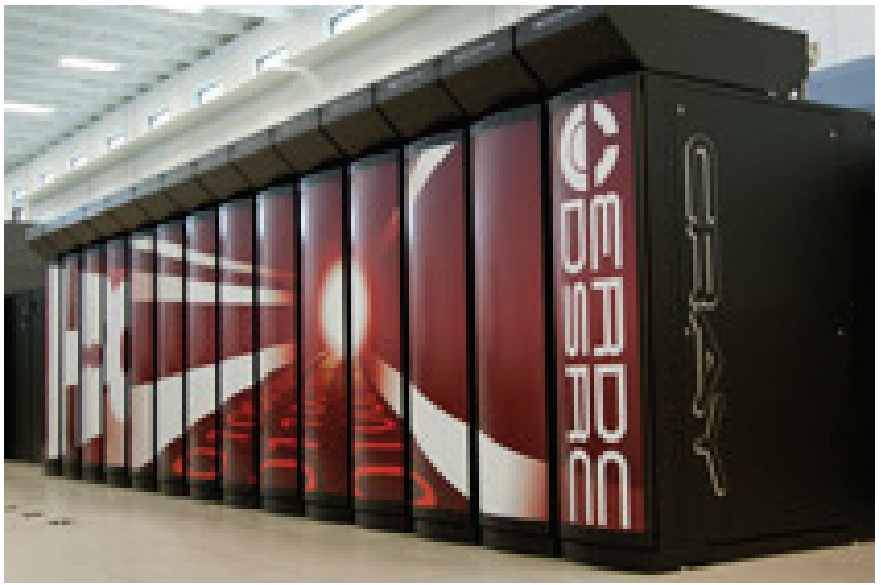




\section{Flow Network Formulation}

The road network offers the most practical example a of flow network. The problem here is to have the maximum amount of traffic flowing through the shortest feasible path. Road networks can be represented as a directed graph to identify the shortest path from point A to point B. In a similar manner, the flow of stresses in a structural system can be interpreted as a directed graph, which facilitates the investigation of questions about failure mechanisms. Intuitively, the flow of information at a given point in the system is the rate at which the information (i.e., variables such as stresses, displacements, kinetic energy, elastic or plastic strain in the structural system) travels. Flow networks can be employed to analyze liquid flow through pipes, parts on assembly lines, current through electrical networks, information through communication networks, flow of energy through food webs in ecosystems, traffic through road networks, etc. (Hiller and Liberman 2004).

Each edge in a directed graph can be compared to a channel through which information is passed. Each edge has a corresponding capacity that is representative of the maximum rate at which information can be passed through the edge (e.g., 100 gallons of liquid per second through a pipe or 11 amperes of electrical current through a wire). Vertices of the graph are the points where the edges connect. All vertices in the graph, except the source and sink, have information flowing through them such that the rate at which information enters the vertex is identical to the rate at which information leaves the vertex. In other words, the flow is conserved in a manner similar to Kirchhoff's Current law, if the flow through the system is electrical current.

Flow networks separate the physics of the problem from the data and map it to an abstract domain where calculations are performed and the solution is mapped back to the physical domain (Tordesillas, Walker and Ando, et al. 2013, Walker and Tordesillas 2010, Tordesillas, Tobin, et al. 2015). Almost any conceivable problem can be solved through the application of flow networks. The basic research described here will develop new methodologies that will provide the foundation for using mathematical algorithms with efficient vector-based programming to tackle highly complex and evolving data obtained from finite element analysis and experiments. To gain a fundamental understanding of superior performance of a biological material, the current research seeks to employ 
flow network analysis. The team used a novel mathematical technique to formulate the rostrum as a network flow problem (Ahuja et al. 1993; Newman 2010). To achieve this, a flow network graph $G=(V, E)$ is developed from the finite element model of the rostrum such that:

1. $V$ Represents the nodes obtained from the finite element model of the rostrum.

2. $E$ Represents the edges, connecting the nodes in $V$, indicating the connectivity between the nodes.

3. Each edge $(u, v) \in E$, has a capacity $C$ associated with it which is representative of the maximum amount of flow that could be transmitted through the edge.

Two nodes are identified in the network to serve as the source $\boldsymbol{s}$ and target $\boldsymbol{t}$ nodes such that the flow can be transmitted from the source node $s$ to the target node $t$. The selection of these nodes will depend on the boundary conditions applied to the rostrum. For example, if the rostrum is subjected to compression loading from the top surface, the source and target nodes can be selected from the top and bottom surfaces, respectively. From Newton's third law of motion it can be inferred that the transmission of flow along each edge is symmetric. Hence, the flow from source to target is identical irrespective of the choice of the source and target nodes.

\subsection{Flow networks and flows}

A flow network is a directed graph $G(V, E)$ consisting of a finite set of vertices together with a subset of ordered pairs of nodes that represent the edges. Each edge $(u, v) \epsilon E$ has a non-negative capacity $c(u, v) \geq 0$. It is assumed that $c(u, v)=0$ if $(u, v)$ does not belong to $E$. Therefore, two nodes are identified in a flow network; a source $\boldsymbol{s}$ and a sink $\boldsymbol{t}$ (Bertsekas 1998; Bondy and Murty 2008; Jungnickel 2008). It is also assumed that every node belongs to a certain path that connects the source and sink (i.e., for each node $v \in V$, there is a path $s \rightarrow v \rightarrow t$ ). In other words, the graph is connected.

A flow in $G$ defined by the real-valued function $f: V \times V \rightarrow R$, is a feasible flow vector if it satisfies the following constraints:

1. Capacity Constraint: $\forall u, v \in V$, requires that $f(u, v) \leq c(u, v)$, the flow cannot exceed the capacity of the respective edge.

2. Conservation of flow: $\forall u, v \in V-\{s, t\}$, requires that 
a. $\sum_{v \in V} f(u, v)=0$

b. The total flow entering a node must equal the total flow leaving that node provided the node is not a source or sink node.

3. The total flow leaving the source node $\boldsymbol{s}$ must be equal to the total flow entering the sink node $\boldsymbol{t}$.

\subsection{Maximum flow in a flow network}

Given a flow network $G(V, E)$, with source $\boldsymbol{s}$ and $\operatorname{sink} \boldsymbol{t}$ such that there are no incoming edges at the source and no outgoing edges at the sink, the maximum flow problem can be defined as follows:

A maximum flow $\boldsymbol{f}$, from source $\boldsymbol{s}$ to sink $\boldsymbol{t}$, is a function that satisfies the capacity and conservation constraints described above and its value is defined as follows:

$$
\operatorname{val}(f)=\max (f)
$$

The maximum flow problem, as described above, can be solved by applying the Ford-Fulkerson algorithm (Bertsekas 1998).

\subsection{Ford-Fulkerson Algorithm}

For a connected graph $G(V, E)$, the flow $f$ in $G$, which is the total flow that leaves the source node $s$ and the total flow that enters the sink node $t$, is defined as follows:

$$
|f|=\sum_{v \in V} f(s, v)=\sum_{v \in V} f(v, t)
$$

The residual capacity of the node pair $(u, v)$, or $c_{f}(u, v)$ is defined as:

$$
c_{f}(u, v)=c(u, v)-f(u, v)
$$

The value of residual capacity is used to determine how much flow can be transmitted between a given set of nodes.

The residual network of $G$ induced by $f$ is the graph $G_{f}=\left(V, E_{f}\right)$ such that

$$
E_{f}=\left\{(u, v) \in V \times V: c_{f}(u, v)>0\right\}
$$

For each edge $(u, v) \in E$ do 


$$
\begin{aligned}
& f[u, v] \leftarrow 0 \\
& f[v, u] \leftarrow 0
\end{aligned}
$$

While $\exists$ a path $p$ from $s$ to $t$ in the residual network $G_{f}$ do

$$
\Delta \leftarrow \min \left\{c_{f}(u, v):(u, v) \in p\right\}
$$

For each edge $(u, v)$ in $p$ do

$$
\begin{gathered}
f[u, v] \leftarrow f[u, v]+\Delta \\
f[v, u] \leftarrow-f[u, v]
\end{gathered}
$$

\subsection{Cuts of flow network}

A cut of a flow network $G(V, E)$ is defined as a set of vertices $\left(E_{1}, E_{2}\right)$ that partition $V$ into $E_{1}$ and $E_{2}=V-E_{1}$ such that $s \in E_{1}$ and $t \in E_{2}$. If $f$ is a flow, then the net flow across the cut $\left(E_{1}, E_{2}\right)$ is defined as $f\left(E_{1}, E_{2}\right)$. The capacity of the cut $\left(E_{1}, E_{2}\right)$ is $c\left(E_{1}, E_{2}\right)$.

A minimum $E_{1}-E_{2}$ cut problem involves minimizing $c\left(E_{1}, E_{2}\right)$. That would mean the identification of $E_{1}$ and $E_{2}$ in such a manner so as to find the minimal capacity of the $E_{1}-E_{2}$ cut.

\subsection{Maximum flow minimum cut theorem}

In combinatorial optimization theory, the maximum flow/minimum cut theorem states that in a flow network $G(V, E)$, the maximum quantity of flow $\boldsymbol{f}$ travelling from the source node $\boldsymbol{s}$ to the sink node $\boldsymbol{t}$ is identical to the total weight of the edges in the minimum cut. Essentially speaking the maximum flow/minimum cut theorem will identify the smallest total weight of the edges which, if removed, would cause the source to be totally disconnected from the sink.

\subsection{Formulate the rostrum as a network flow problem}

The output from the finite element model will be input to the network flow algorithm to gain insight into the load transfer mechanism and formation of the stress distribution pattern in the network. Mathematical techniques are needed to understand how these patterns are formed at the onset of the 
external loading. For fractal and discrete systems, flow patterns in the networks are known to be the key for load transmission. When the networks are short circuited, deformation ensues. Mathematical techniques have been used to examine interactions within various complex systems such as traffic flow, energy flow through food webs in an ecosystem, fluid flow through pipelines, communication networks, electrical networks, community structure of company ownership, patrol routing problem, airtransportation, failure of quasi-brittle materials, and force transmission in dense granular media (Schrijver 2002; Vitali et al. 2011; Barrat et al. 2004; Bullmore and Sporns 2009; Dewil et al. 2015; Lefort et al. 2015; Lin and Tordesillas 2014; Duran 2000; Radjai et al. 1998; Tordesillas 2007; Tordesillas et al. 2012). However, to our knowledge, advanced mathematical techniques have not been used to study the complex geometrical hierarchical configuration and structural response of living specimens.

The authors elucidate the working of the proposed flow network approach through a toy network constructed from a small region of the rostrum as shown in Figure 16. For a given flow network, the quantity of maximum flow is equal to the capacity of the minimum cut (Cormen et al 1990). By using the Ford-Fulkerson algorithm described above, the capacity of the minimum cut is determined. For example, for the flow network shown in Figure 16, the possible cuts are $\{(0-1),(0-5)\},\{(9-8),(6-8),(8-12)\},\{(5-6)\}$ etc. The minimum source-target cut is $(5-6)$ which has a capacity of 26 (highlighted in red in Figure 16). Based on the flow patterns (governed by stresses, strains, or deformations) established at the onset of the load application, the flow network approach can aid in detecting the failure site much earlier (i.e., before the material fails or when the material is in the linear elastic regime of the stress strain curve) than any computational or analytical methodologies. The current study uses the output from the numerical solution for the rostrum of the paddlefish as input to the flow network algorithm. Therefore, a parallel algorithm is developed to provide the relevant data to the flow network algorithm. This in-house written algorithm extracts the connectivity information from the numerical model; weights (Von-Mises stresses in this case) are extracted for the output database file produced by the numerical model. The weights are obtained from the dynamically evolving complex numerical analysis data. 
Figure 16. A demonstration model constructed from a small part of the rostrum to illustrate the modus operandi of maximum flow-minimum cut (MFMC) algorithm.

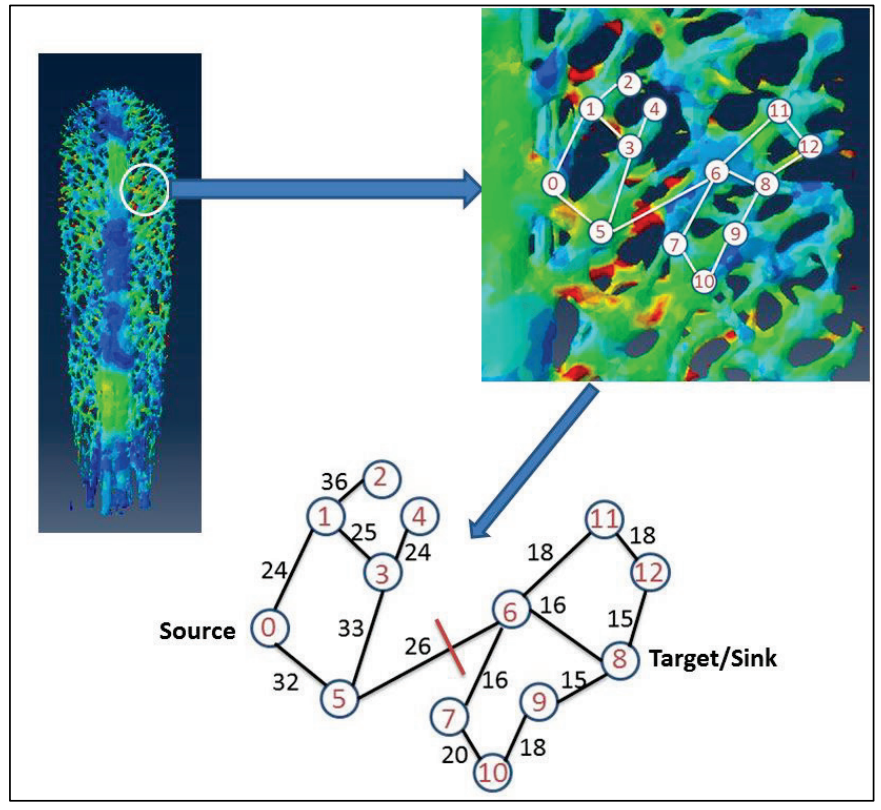




\section{Results}

Computational mechanics experiments are conducted on the rostrum model using Abaqus to analyze the yielding and load transfer mechanisms. Based on the Von-Mises stresses, the load transfer mechanism and yielding process of the different components of the rostrum are investigated.

Although computational mechanics experiments provide details of the overall stress distribution in the system, the underlying details of the load transfer mechanisms of the systems is still missing. These mechanisms play a vital role in formation of stress distribution pattern governed by major load bearing members of the structural systems. Most bio-inspired structures are designed for mechanical strength/resilience and utilize solid, dense materials and uniform pattern in construction reflecting the construction of their hard, heavy biological models: ram horns, turtle shells, and abalone valves. This study will describe a structure that has been optimized for mechanical strength/resilience and for hydrodynamic efficiency, which utilizes soft, lightweight elements and a non-uniform pattern in construction: the cartilage lattice, of variable pore size, comprising the endoskeleton of the Paddlefish rostrum. Towards this end, the surface topology obtained from micro CT imagery and weighting function based on strain and flow measurement, which are thought to be the most reliable data that can be measured, will be used to identify the patterns. The pattern recognition will be used to correlate for the Early Detection of Failure Mechanisms in Resilient Biostructures.

\subsection{Test case details}

The uniform pressure loading experiment on the rostrum model is performed using the general static analysis available in Abaqus/Standard*. The choice of Abaqus/Standard for performing these analyses is justified based on the solution technology that it employs for static and low-speed dynamic events wherein highly accurate stress solutions are required.

All three components of the rostrum, namely the tissue, soft cartilage, and hard cartilage, are modeled using the material properties of steel as discussed in section 2.2 to study the response of rostrum to a uniform

* Acosta, F. J., G. A. Riveros, R. R. Patel, and W. Hodo. In prep. Numerical simulation of biological structures: Paddlefish Rostrum. ERDC Technical report. 
pressure loading. The variation of the stress distribution in the three components of the rostrum with increasing pressure load is extracted from the finite element solution as the components part yields.

The rostrum is formulated as a network flow model by obtaining the node and connectivity information from the computational mechanics model. Maximum flow/minimum cut algorithms will be used to identify the patterns when rostrum is subjected to external loads. The pattern recognition may be used to correlate for early detection of failure mechanisms in resilient Biostructures.

\subsection{Stress distribution in the rostrum subjected to uniform pressure}

The stress distribution based on the Von-Mises stresses is analyzed from the output database files for the rostrum subjected to the uniform pressure loading condition* ${ }^{*}$. The Von Mises stresses are equivalent tensile stresses derived from the stress deviator (Segalman et al. 1998). An upper limit of $345 \mathrm{MPa}$ for the yield stress is set for the Abaqus stress contour plots. Any element that exceeds a value of $345 \mathrm{MPa}$ is colored grey. The value of 345 $\mathrm{MPa}$ is chosen because all components of the rostrum are modeled as steel as stated in section 2.2 earlier and it corresponds to the yield strength of steel used in the computational mechanics experiments conducted in the current study. Figure 17 shows the Von-Mises stresses for the three components of the rostrum. As seen in Figure 17, as the uniform pressure on the rostrum reaches a value of 9.5 $\mathrm{MPa}$, the elements of the tissue start yielding. The hard and soft cartilage elements do not show any yielding at this stage. As the pressure increases, yielding is observed in the elements of the hard cartilage when the uniform pressure reaches a value of 18.5 $\mathrm{MPa}$. At this point, the soft cartilage elements do not show any yielding. As the pressure increases to 27.5 $\mathrm{MPa}$, the soft cartilage elements start yielding. The load is transferred from the outer soft layer of the tissue to the inner hard cartilage and then to the innermost soft cartilage as the pressure increases.

\footnotetext{
* Acosta, F. J., G. A. Riveros, R. R. Patel, and W. Hodo. In prep. Numerical simulation of biological structures: Paddlefish Rostrum. ERDC Technical report.
} 
Figure 17. Contour plot of Von-Mises Stresses for the three components of rostrum. Yielding regions are circled for clarity*.

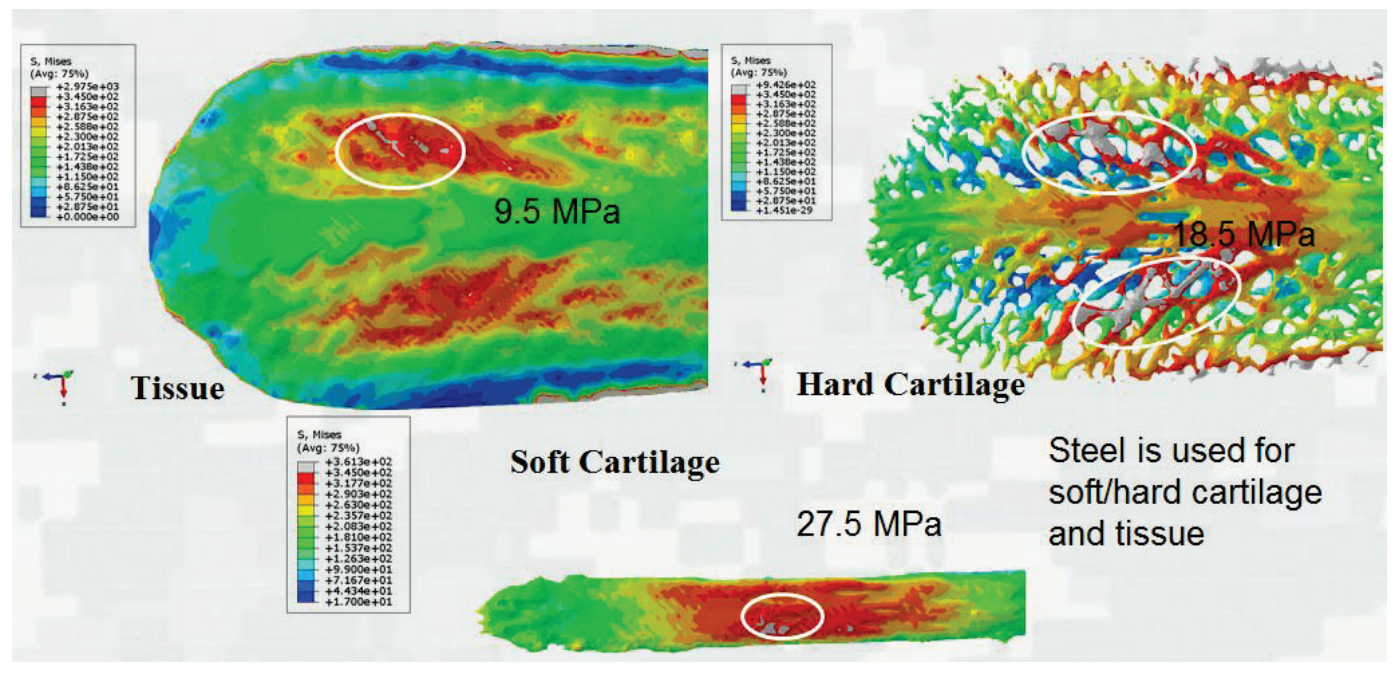

\subsection{Stress distribution in the rostrum subjected to uniform pressure: tissue}

The tissue component of the rostrum not only includes the exterior region of the rostrum but also encompasses the material that encases the hard and soft cartilage. Since it is the outermost region, its elements are in direct contact with the pressure loading. As seen in Figure 18, at a uniform pressure of 9.5 $\mathrm{MPa}$, no elements in the tissue show yielding. As the uniform pressure load increases to a value of 18.5 MPa, the tissue elements start yielding. The elements that have yielded are colored grey. The tissue elements progressively yield as the pressure increases from 27.5 MPa to 50.0 MPa. The yielding starts in the region where the rostrum is thinnest as expected. Also, the part of the rostrum attached to the mouth of the fish does not yield even when the pressure is 50.0 MPa.

\footnotetext{
* Acosta, F. J., G. A. Riveros, R. R. Patel, and W. Hodo. In prep. Numerical simulation of biological structures: Paddlefish Rostrum. ERDC Technical report.
} 
Figure 18. Stress distribution due to uniform pressure loading on rostrum: tissue.*
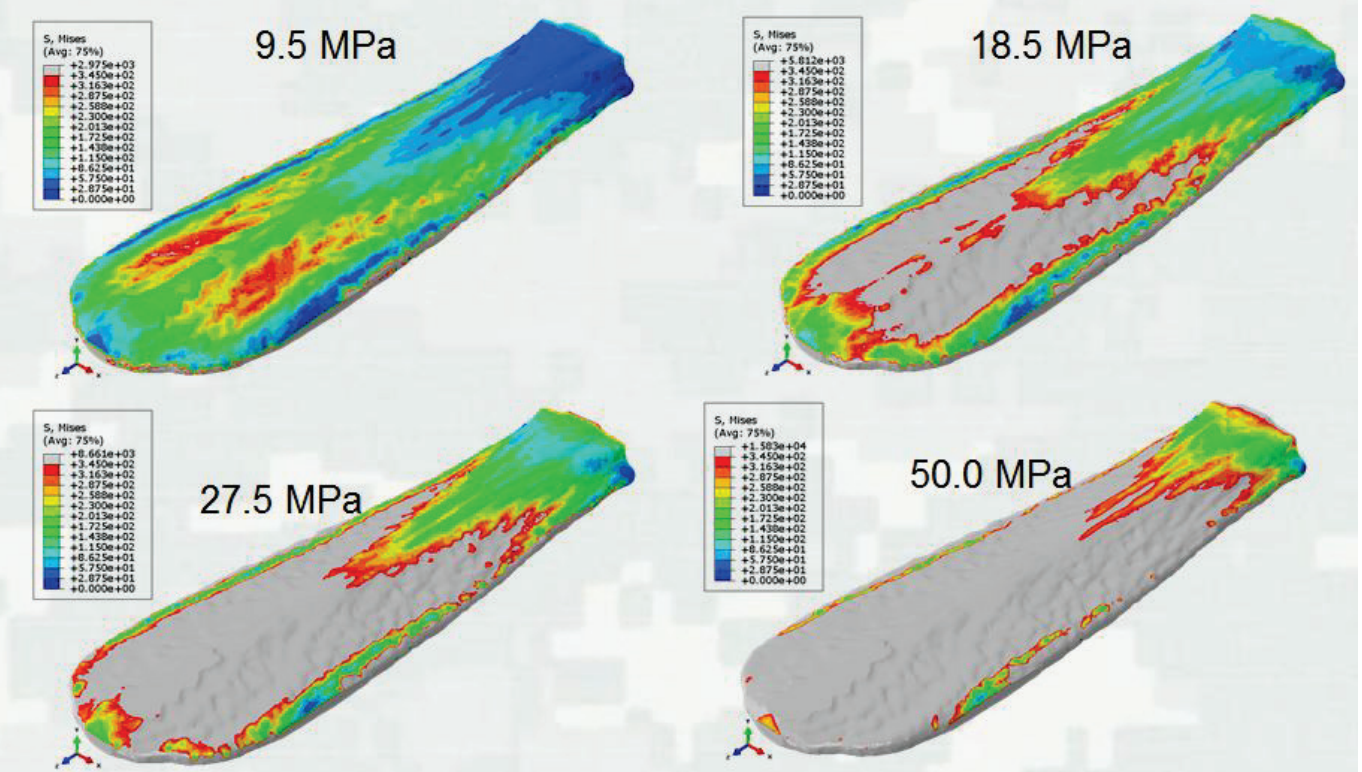

\subsection{Stress distribution in the rostrum subjected to a uniform pressure: hard cartilage}

The hard cartilage is formed by two flat sections that are almost parallel to each other and run along the longitudinal axis of the rostrum and branch out from the center to the full width of the rostrum forming a truss-like structure. The elements of the hard cartilage do not show any yielding when the uniform pressure is 9.5 $\mathrm{MPa}$. When the pressure increases to 18.5 MPa, few elements show yielding as represented in Figure 19. The yielding in the hard cartilage elements at 18.5 MPa pressure is not as widespread as that in the tissue elements as seen in Figure 18. The elements of the hard cartilage progressively yield as the pressure increases from 27.5 MPa to 50.0 MPa. As stated earlier, the yielding of hard cartilage elements is not as significant at that for tissue elements at similar pressures.

\footnotetext{
* Acosta, F. J., G. A. Riveros, R. R. Patel, and W. Hodo. In prep. Numerical simulation of biological structures: Paddlefish Rostrum. ERDC Technical report.
} 
Figure 19. Stress distribution due to uniform pressure loading on rostrum: hard cartilage*.

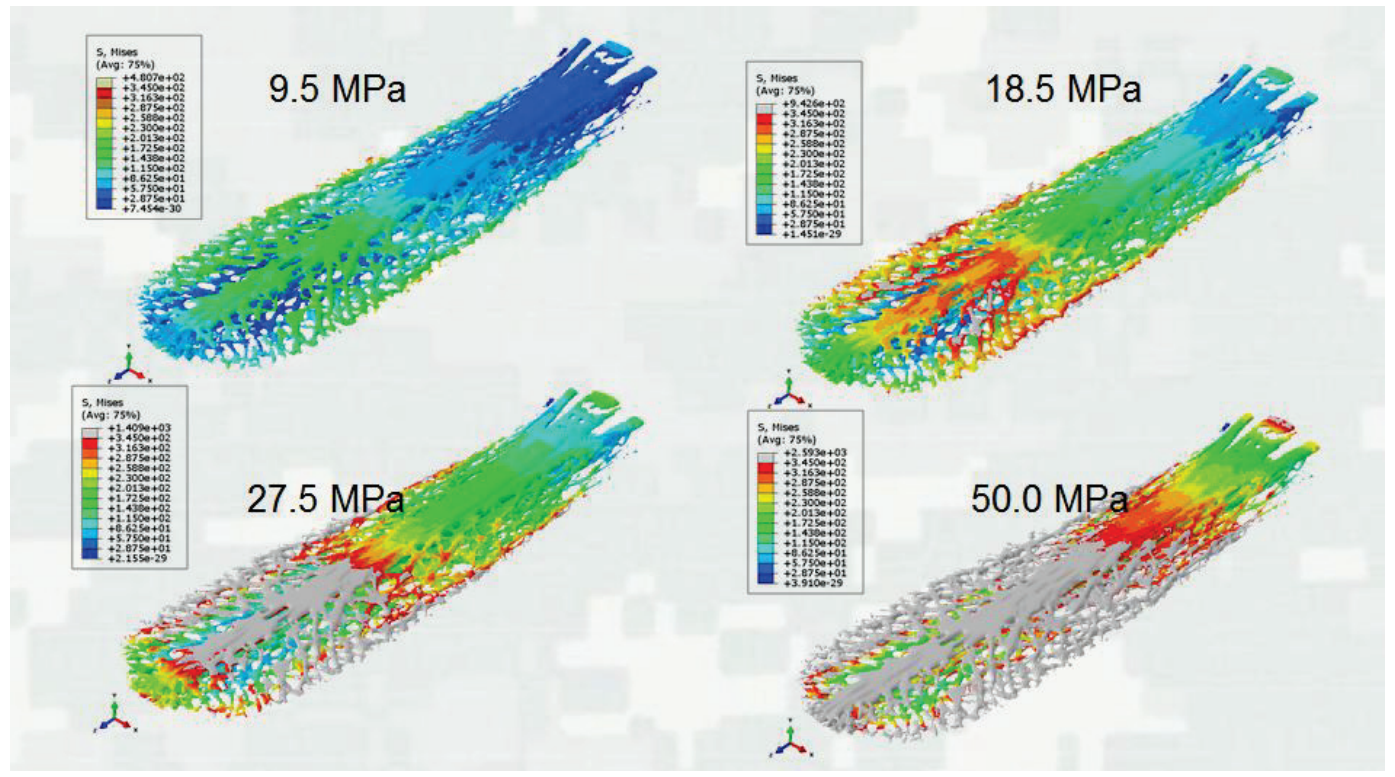

\subsection{Stress distribution in the rostrum subjected to a uniform pressure: soft cartilage}

The soft cartilage is located at the center of the rostrum and runs in the longitudinal Z-direction. As seen in Figure 20, the soft cartilage elements do not show any yielding until the uniform pressure load reaches 27.5 MPa. The elements of the soft cartilage yield progressively as the uniform pressure increases from 27.5 MPa to 50.0 MPa. The elements of the soft cartilage show comparatively less yielding at 50.0 MPa than the elements of the hard cartilage and tissue at 50.0 $\mathrm{MPa}$.

\footnotetext{
* Acosta, F. J., G. A. Riveros, R. R. Patel, and W. Hodo. In prep. Numerical simulation of biological structures: Paddlefish Rostrum. ERDC Technical report.
} 
Figure 20. Stress distribution due to uniform pressure loading on rostrum: soft cartilage.

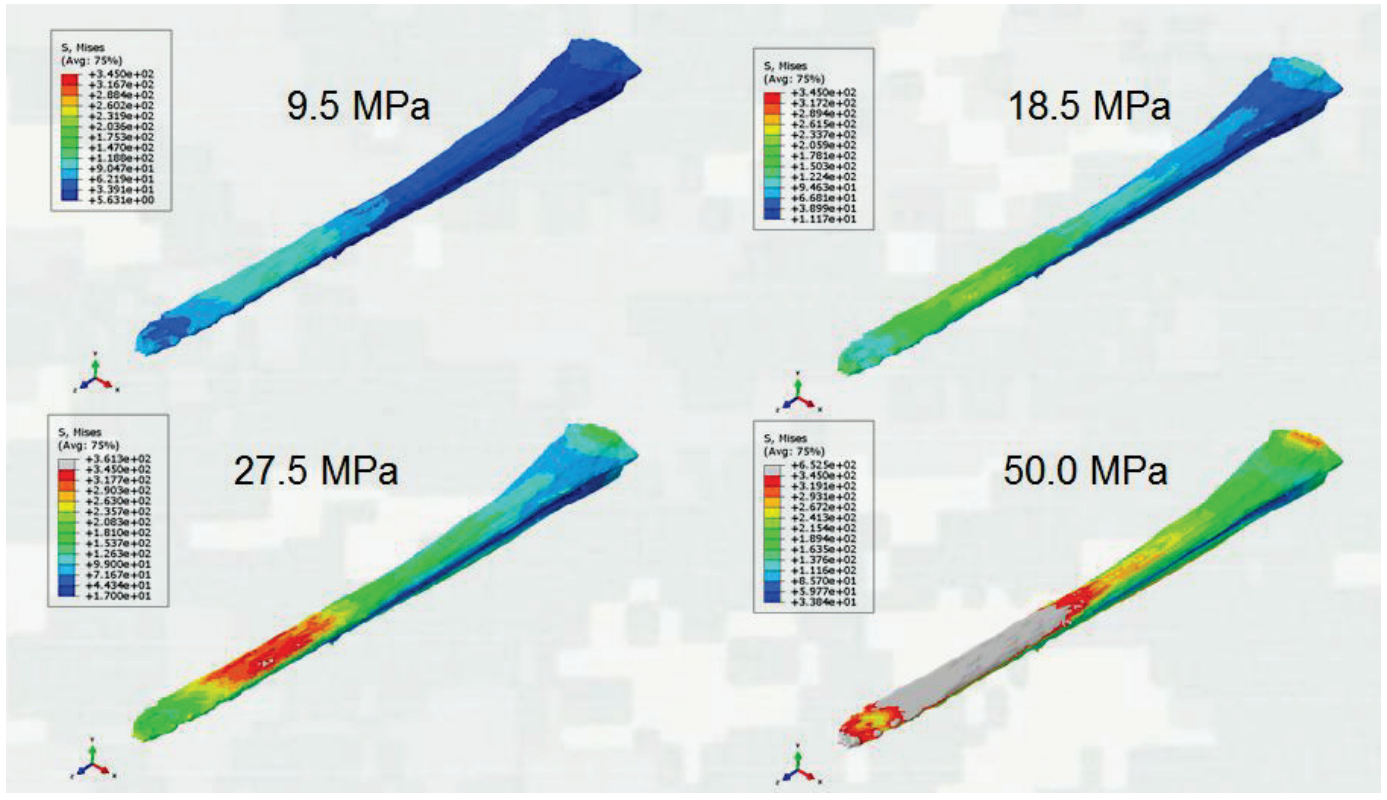

\subsection{Network flow analysis of the load distribution with increasing pressure on rostrum}

Although the computational mechanics experiments on the rostrum gives a clear picture of how the load is transferred from the tissue to the hard cartilage to the soft cartilage as the uniform pressure load on the rostrum increases, it remains unclear how the stress distribution patterns are formed at the nascent stage of loading. Also, the computational mechanics experiments on a complex geometry will involve a high degree of freedom thereby making the extraction of these finer details computationally expensive (i.e., identification of which members bear the maximum load and which members provide support to the load bearing members). These intricate details, which play a vital role in failure mechanisms and stress distribution pattern in the network, are still unknown.

As outlined in section 3.6 earlier, the rostrum model is formulated as a network flow problem. The nodes and connectivity information are extracted from the finite element model of the rostrum. The weights of the network are obtained from the Von-Mises stresses in the output database file produced from the computational mechanics experiments for the rostrum. The information extracted from the computational mechanics experiments is input to the maximum flow algorithms described in (Boykov and Kolmogorov 2004). A seamless platform-independent interface is developed to extract the flow network connectivity and weight 
information from the output database files produced from the computational experiments on the rostrum.

The source and sink are identified and explicitly input to the maximum flow/minimum cut algorithm. Figure 21 shows the output from the maximum flow/minimum cut algorithm. The red region is representative of the formation of force chains in the rostrum under the influence of an external loading condition (in this case, a uniform pressure loading on upper surface of the rostrum). Initially, when the pressure is $1.428 \mathrm{MPa}$, the force chains are formed in the lower left region of the rostrum. This asymmetrical behavior can likely be attributed to the unsymmetrical geometry of the rostrum. As the pressure increase to $2.857 \mathrm{MPa}$, force chains are formed in the lower left and right regions of the rostrum. Also, as the pressure increases, stress localizations are seen near the center bone of the rostrum. This is indicative of the region being under tension. Also, there is no transmission of information on the top portion of the rostrum indicative of the fact that the rostrum is comparatively thinner in that region and also prone to failure. The force chains are populated in the bottom region of the rostrum where it is attached to the mouth of the fish. Also, from the computational mechanics simulations, it is clear that this region of the rostrum did not yield even at the maximum applied pressure of $50 \mathrm{MPa}$. Also, since the rostrum is thicker near the mouth of the fish, it is able to withstand higher loads in comparison to the upper, thin region.

Figure 21. Network flow analysis of load distribution with increasing pressure on rostrum's bottom surface.
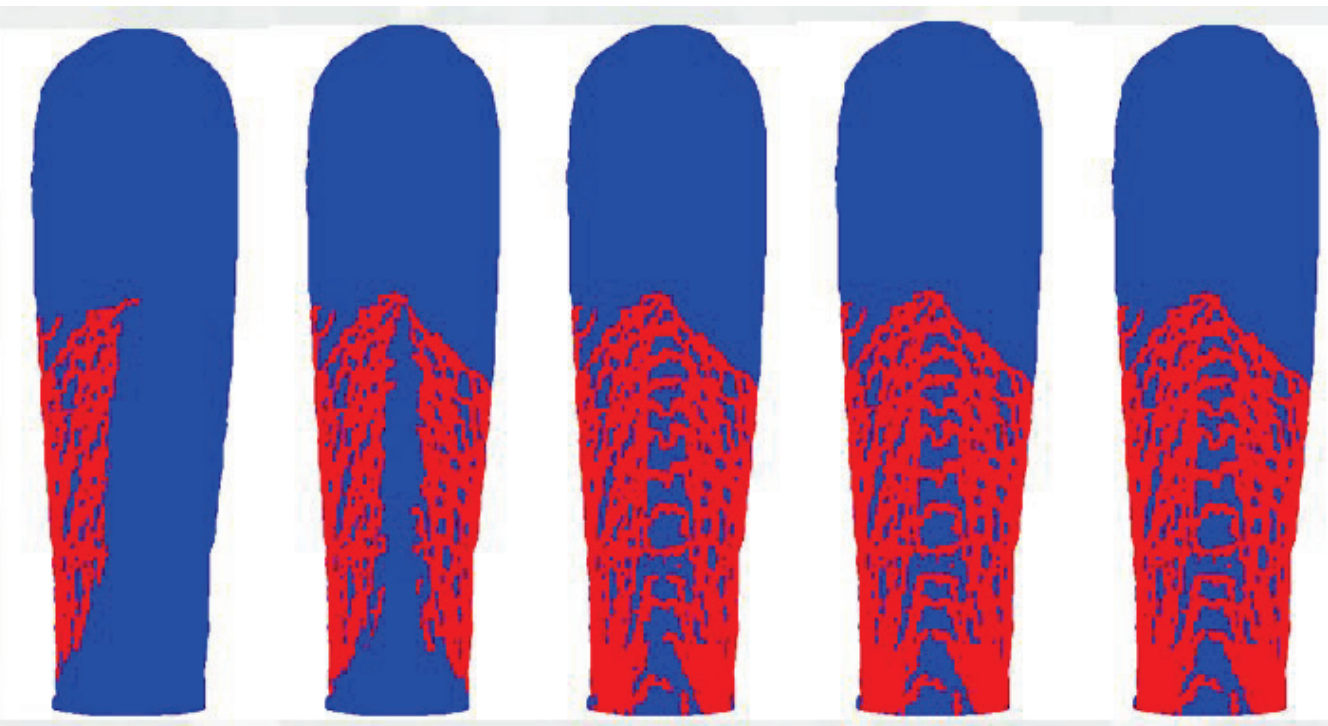

$1.428 \mathrm{MPa} \quad 2.857 \mathrm{MPa}$

$4.285 \mathrm{MPa}$

$5.714 \mathrm{MPa}$

$7.143 \mathrm{MPa}$ 
Network flow analysis using the maximum flow/ minimum cut algorithm on the top surface of the rostrum is depicted in Figure 22. At a uniform pressure of $1.428 \mathrm{MPa}$, the lower right portion of the rostrum shows the formation of force chains. This asymmetrical distribution of load is again attributed to the unsymmetrical geometry of the rostrum. As the pressure increases to $2.857 \mathrm{MPa}$, force chains start forming on the lower left portion of the rostrum. With further increase in pressure, more force chains are formed on the lower portion of the rostrum. The top portion of the rostrum shows a similar behavior to a beam under a compression loading condition. Force chains are not formed on the top portion of the rostrum, which is thinner as compared to the bottom portion of the rostrum. The part of the rostrum near the mouth of paddlefish shows the largest force chain formation, indicative of it being stronger than the top portion of the rostrum, which is the thinnest.

Figure 22. Network flow analysis of load distribution with increasing pressure on rostrum's top surface.

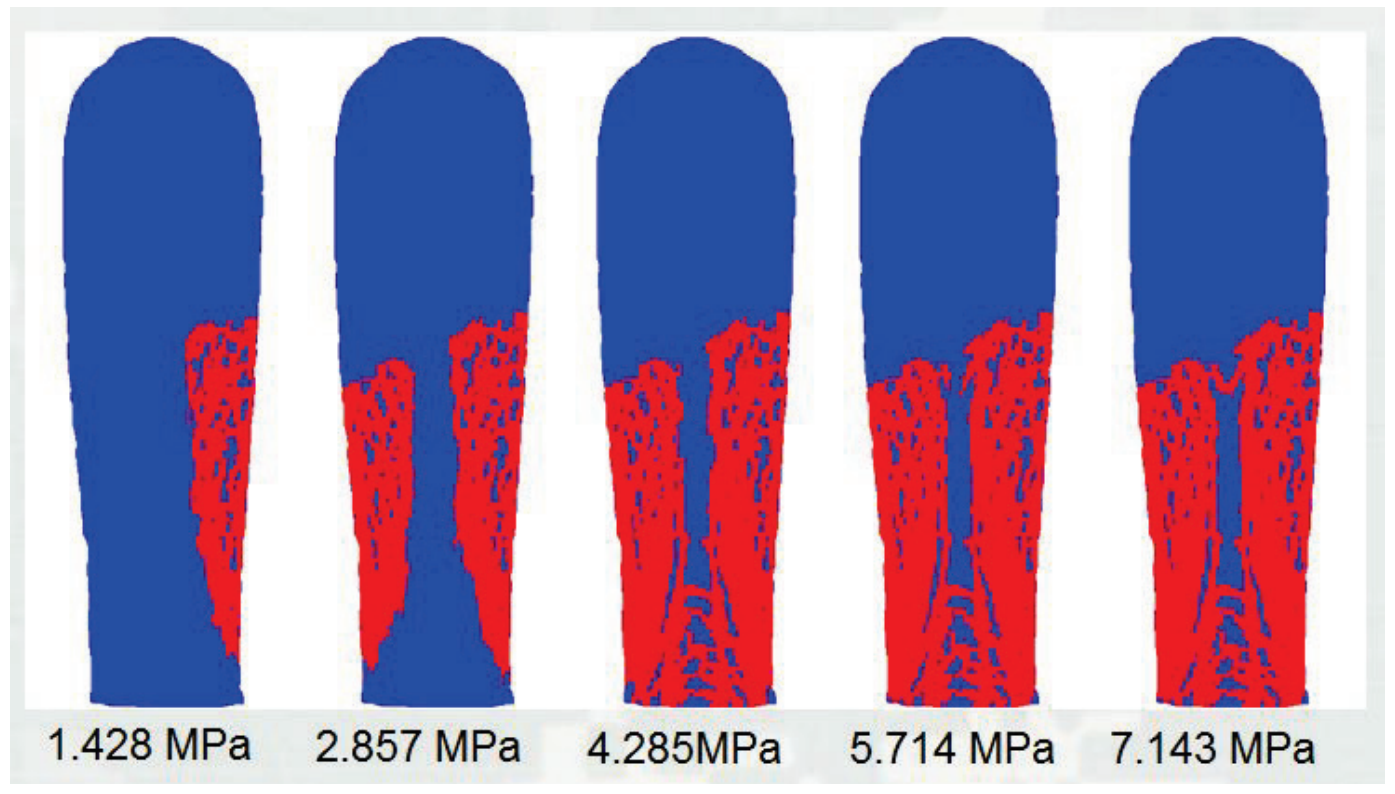




\subsection{Stress distribution in the rostrum without the lattice architecture}

To analyze the effect of the complex lattice architecture of the hard cartilage of the rostrum, a model of the rostrum was constructed without the lattice architecture. Figure 23 shows the computational model of the rostrum that was constructed in Abaqus. A homogenous material property (assumed to be steel) was assigned to this model. This model had the geometry of the rostrum's outer layer but lacked the intricate detail of the hard and soft cartilage regions of the rostrum. This model consisted of 159,716 elements and 32,402 nodes.

Figure 23. Rostrum without lattice architecture.

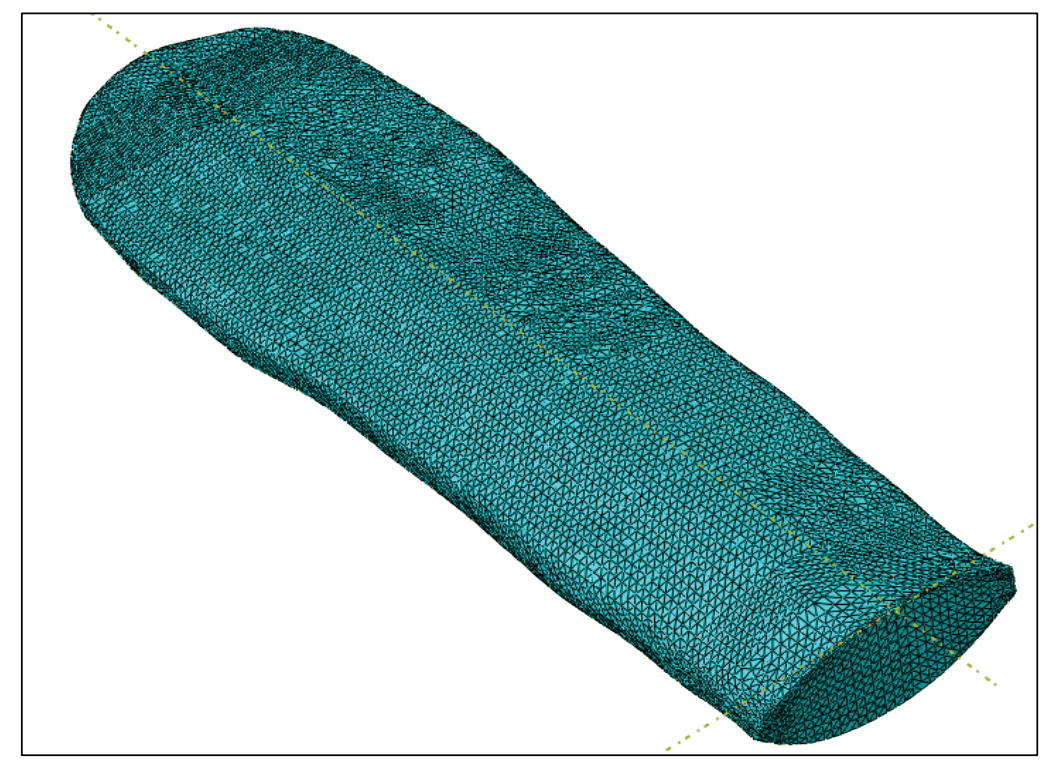

\subsection{Network flow analysis of the load distribution with increasing pressure on the rostrum without the lattice architecture}

A flow network model of the homogenous rostrum displayed in Figure 23 is constructed from the connectivity and node information from the computational mechanics model. This analysis is conducted to study the response of the rostrum with and without lattice architecture. The rostrum model experienced comparatively more stress in absence of the lattice architecture. Also, the maximum flow /minimum cut algorithm revealed that highly localized force chains are formed in the absence of the lattice architecture (Figure 24). Biostructures perform better as compared to man-made materials because of the presence of such hierarchical geometries in their architecture. Localized force chains make the rostrum 
without the lattice architecture more prone to failure as well as experiencing higher stresses.

Figure 24. Network flow analysis of load distribution with increasing pressure on rostrum without lattice architecture.

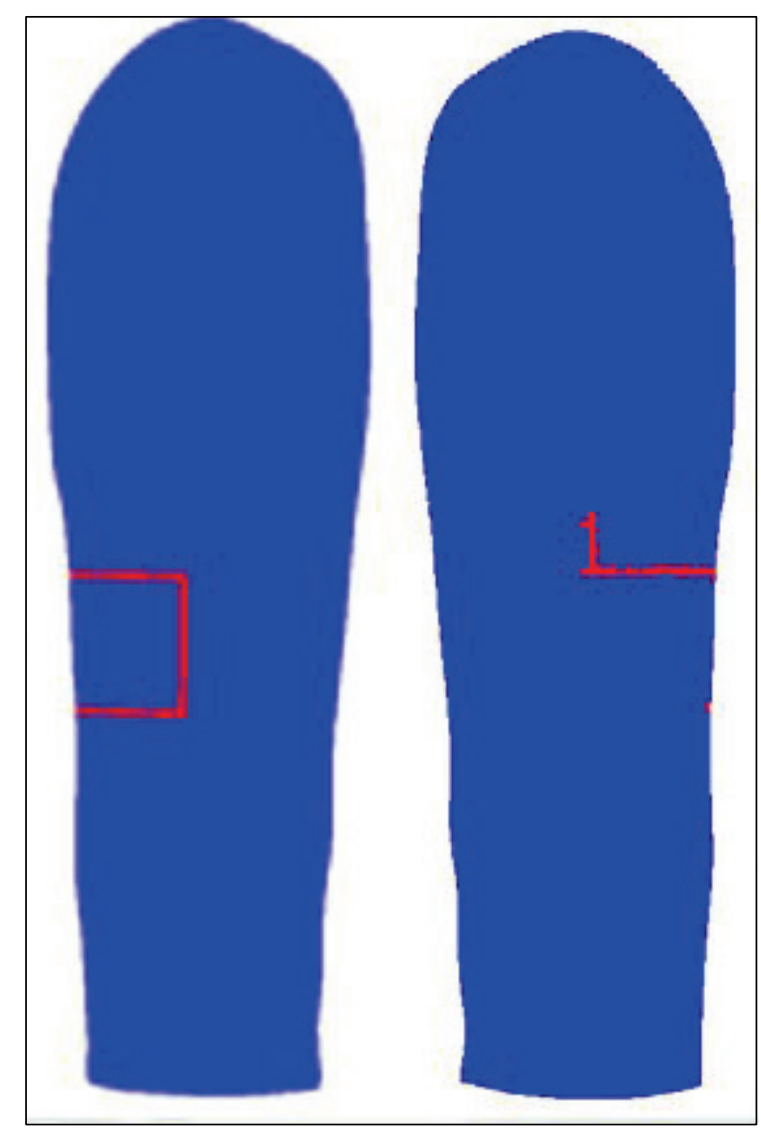

\subsection{Analytical verification of methodology}

The customary technique for verifying a simulation is to employ a problem that is simple enough to have an analytical solution and similar enough to the phenomena that is being simulated so that a meaningful extrapolation to the actual problem is feasible. Following this procedure enables validating the numerical solution or methodology with an exact, analytically computed solution to the simplistic problem. By doing so, one can compare the numerical and analytical solutions and identify fundamental shortcomings with either the methodology or the numerical approach used to solve the problem.

To verify the flow network approach used in the current research, the problem of a simply supported beam with a concentrated load at the 
center was chosen. The schematic of the beam with this loading condition and the shear and moment diagrams are represented in Figure 25 (Gere and Timoshenko 1996). Abaqus was used to perform the computational mechanics experiment on a concrete beam of length 216 inches, width 36 inches, and thickness 6 inches. A mass density of 8.67e-011 slug/in3, Young's modulus of $3 \times 10^{6} \mathrm{MPa}$, and Poisson's ratio of 0.3 was used for the concrete material model. The Abaqus mesh used for validation model consisted of 972 linear hexahedral elements as shown in Figure 26. The Von-Mises stresses obtained from the computational mechanics experiment are plotted in Figure 27, which gives the overall stress distribution in the beam subjected to a concentrated load of $100 \mathrm{MPa}$ at the center. The simulation was carried out using the dynamic, explicit method in Abaqus for a total time of $0.005 \mathrm{~s}$. Geometric non-linearity effects were included. The node and connectivity information were extracted from the computational mechanics experiments and a flow network was constructed. The network was weighted based on the VonMises stress values from the Abaqus output database file. The result of the network flow analysis is shown in Figure 28. The flow network highlights the members of the beam that take the maximum load. Also, as seen from Figure 25, the maximum moment is at the center of the beam. Hence, the proposed methodology for recognizing the failure region from the computational mechanics simulation and flow network approach is validated using a simple problem with a known solution. 
Figure 25. Simply supported beam with concentrated load at the center.

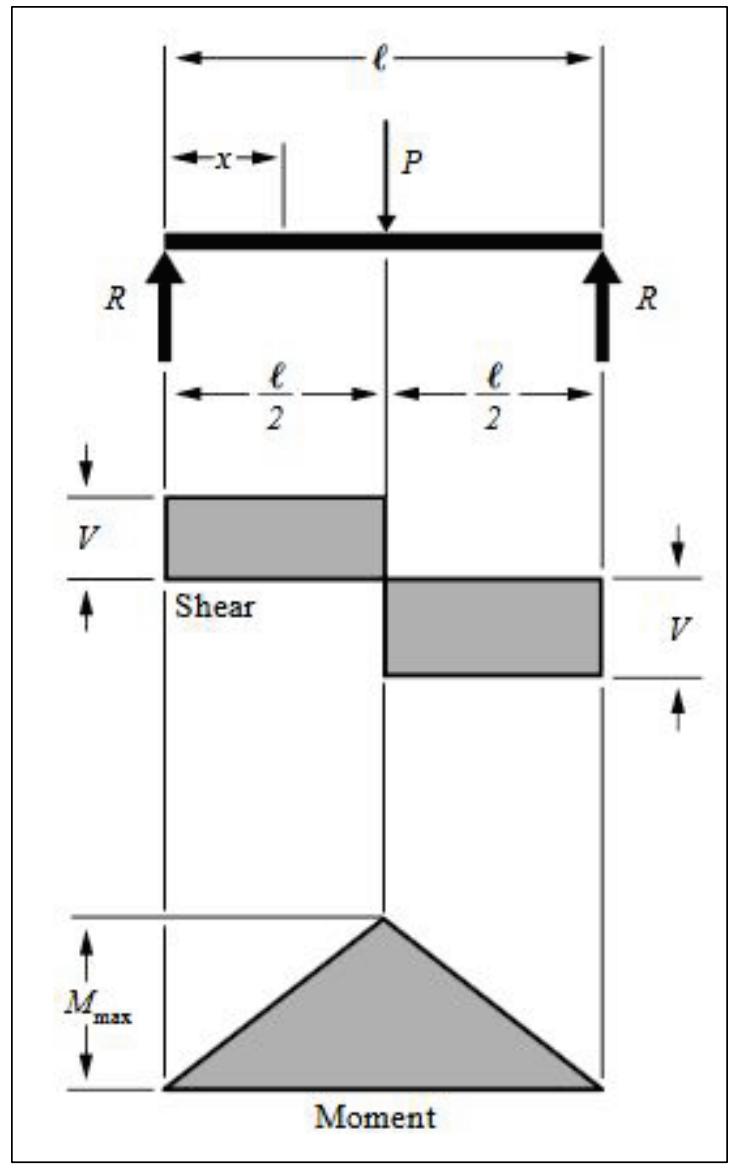

Figure 26. Abaqus mesh.

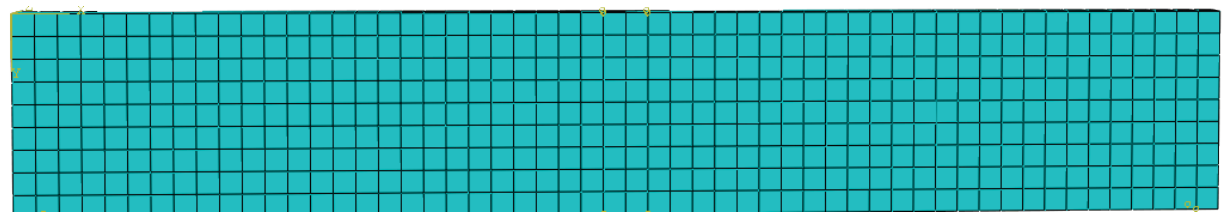


Figure 27. Von-Mises stresses.

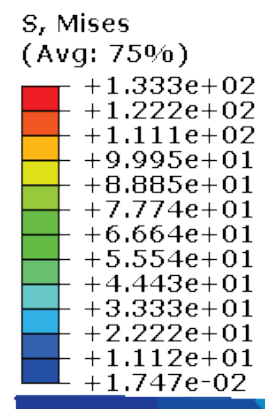

Figure 28. Flow network result. 


\section{Conclusions and Recommendations}

\subsection{Conclusions}

Biostructures are highly complex because of the hierarchical geometrical arrangement and heterogeneous material properties. The structural response of Biostructures is hard to predict owing to this complexity. Although computational mechanics experiments on the rostrum reveal the load transfer mechanisms and the overall stress distribution for a uniform, external loading, the underlying kinematics that plays a vital role in the load transfer mechanisms and the formation of the strong and weak links in the networks is still unknown. The approach presented in this research is a means to identify the patterns of force chain formation and identification of the load bearing members through efficient application of the maximum flow/minimum cut algorithm. The force chain formation when the structural system is under the influence of the external uniform pressure is clearly depicted. The center bone stiffens the system while the surrounding complex bone structure is an efficient load transferring system.

\subsection{Load transfer mechanisms for the rostrum}

Computational mechanics experiments on the rostrum revealed that the outermost layer of the rostrum, (i.e., the tissue), is in direct contact with the external loading condition and hence the elements of the tissue yield first. The inner hard and soft cartilage components of the rostrum do not show any yielding at lower pressures. As the pressure increases, the yielded elements of the tissue transfer the load to the inner hard cartilage. When the hard cartilage elements yield, the load is transferred to the innermost soft cartilage layer. This load transfer mechanism in the rostrum provides excellent energy dissipation. With the complex lattice architecture, the rostrum experienced comparatively less stress than tissue without the lattice when subjected to an identical external loading condition. The complexity of the hierarchical geometrical configuration and the heterogeneous materials found in Biostructures makes them lightweight, strong, and gives them better energy dissipation characteristics.

\subsection{Stress distribution patterns in the rostrum}

In this study, the maximum flow optimization problem and the maximum flow/minimum cut pathways for a general flow network that was 
formulated from the node and connectivity information of the rostrum under a uniform pressure loading condition have been considered. This objective was to examine the stress distribution pattern in the flow network that were weighted based on the stresses experienced by the structural system. The rostrum's lattice architecture is a major contributor to its superior performance. The present study conducted a mathematical analysis on rostrum with and without the lattice architecture and found highly localized stress distribution patterns in the rostrum in the absence of the lattice architecture. The lattice is considered an indeterminate, nonlinear structure with varying material types, and material properties, with non-uniform stiffness and irregularly-shaped members. The maximum flow /minimum cut approach helped in the analysis of the indeterminate lattice architecture. The flow network was able to predict the strain localization in the tensile region of the rostrum. Also, the flow network was able to identify the asymmetrical response of the rostrum to uniform loading.

\subsection{Future work recommendation}

1. The current study analyzed the effect of the absence of the lattice architecture and its effect on the stress distribution and force chain formation in the rostrum. Highly localized stress distribution patterns were observed in the rostrum in the absence of the lattice architecture. Using different lattice architectures and correlating the complexity of the lattice with the strength and resilience of the rostrum for varying loading condition will provide better insight into the role that geometry plays in achieving structural resiliency.

2. The rostrum model obtained from multiple CT-scans used in the study was a surface mesh. The geometry of the rostrum was not available to conduct this study. Future work will involve the mesh refinement study and its effect on the results.

3. The approach used in the present work was verified using analytical solution to a simple problem. Physical experiments will be conducted on the rostrum with cantilever beam boundary condition and also measuring the stress and performance of the rostrum in a live swimming paddlefish for a more complete validation.

4. Rostrums from different stages of the life of paddlefish, namely juvenile, sub-adult, and adult, will be analyzed to see the effects of varying the rostrum's lattice architecture. This will aid in determination of how increasing geometrical complexity in lattice networks contributes to mechanical performance. 


\section{References}

Ahuja, R. K., T. L. Magnanti, and J. B. Orlin. 1993. Network flows theory, algorithms, and applications. London: Pearson.

Allen, J. B., and G. A. Riveros. 2013. Hydrodynamic characterization of the polyodon spathula rostrum using CFP. Journal of applied mathematics. 2013. http://dx.doi.org/10.1155/2013/346173.

Allis, E. P., Jr. 1903. On certain features of the lateral canals and cranial bones of Polyodon folium. Zoologische Jahrbucher 17:659-678.

Allison, P. G., M. Q. Chandler, R. I. Rodriguez, B. A. Williams, R. D. Moser, C. A. Weiss, A. R. Poda, B. J. Lafferty, A. J. Kennedy, J. M. Seiter, and W. D. Hodo. 2013. Mechanical properties and structure of the biological multilayered material system, Atractosteus spatula scales. Acta Biomaterialia 9(2):5289-5296.

Barrat, A., M. Barthelemy, R. Pastor-Satorras, and A. Vespignani. 2004. The architecture of complex weighted networks. In Proceedings of the National Academy of Sciences of the United States of America 101(11):3747-3752.

Bertsekas, D. P. 1998. Network optimization: Continuous and discrete models (optimization, computation, and control). Belmont: Athena Scientific.

Bondy, J. R., and U. S. R. Murty. 2008. Graph theory. New York: Springer.

Boykov, Y., and V. Kolmogorov. 2004. An experimental comparison of min-cut/max-flow algorithms for energy minimization in vision. IEEE Transactions on Pattern Analysis and Machine Intelligence (PAMI) 26(9): 1124-1137.

Bruce, D. W., and D. O'Hare. 1996. Inorganic materials. London UK: John Wiley \& Sons.

Bullmore, E., and O. Sporns. 2009. Complex brain networks: graph theoretical analysis of structural and functional systems. Nature Reviews. Neuroscience 10(3):186-198.

Cormen, T. H., C. E. Leiserson, and R. L. Rivest. 1990. Introduction to Algorithms. The MIT electrical engineering and computer science series. Cambridge: McGraw Hill.

Davis Jr., P. W. 1959. The yellow bodies of the condrostean fish, Polyodon spathula. Bios 30(3) $155^{-158 .}$

Deang, J., M. Horstemeyer, L. Williams, E. Perkins, P. Allison, and G. Riveros. 2016. Paddlefish rostrum as a structure for bioinspiration: Analysis and modeling of the of the stress state and strain rate vependence behavior of cartilage. TMS Annual Meeting \& Exhibition.

Dewil, R., P. Vansteenwegen, D. Cattrysse, and D. Van Oudheusden. 2015. A minimum cost network flow model for the maximum covering and patrol routing problem. European Journal of Operational Research 247(1):27-36. 
Duran, J. 2000. Sands, powders, and grains: An introduction to the physics of granular materials. New York: Springer Science \& Business Media.

Gere, J. M., and S. P. Timoshenko. 1996. Mechanics of materials: Fourth Edition. PWS Publishing Company.

Grande, L., and W. E. Bemis. 1991. Osetology and phylogenetic relationships of fossil and recent paddlefishes (Polyodontidae) with comments on the interrelationships of Acipenseriformes. Journal of Vertebrate Paleontology. 11(S1):1-120.

Hiller, F. S., and G. J. Liberman. 2004. Introduction to Operations Research. Eighth edition. New York: McGraw-Hill.

Hoover, J. J., S. G. George, and K. J. Killgore. 200o. Rostrum size of paddlefish (Polyodon spathula) (Acipenseriformes: Polyodontidae) from the Mississippi delta. Copeia 2000(1):288-290.

Hoover, J. J., K. A. Boysen, C. E. Murphy, and S. G. George. 2009. Morphological variation in juvenile Paddlefish. In American Fisheries Society Symposium 66: 157-171.

Jungnickel, D. 2008. Graphs, networks and algorithms.Third edition. Berlin GE: Springer Berlin.

Lefort V., G. Pijaudier-Cabot, and D. Gregoire. 2015. Analysis by Ripley's function of the correlations involved during failure in quasi- brittle materials: experimental and numerical investigations at the mesoscale. Engineering Fracture Mechanics 147:449-67.

Lin, Q., and A. Tordesillas. 2014. Towards an optimization theory for deforming dense granular materials: Minimum cost maximum flow solutions. Journal of industrial and management optimization 10(1): 337-362.

Newman, M. E. J. 2010. Networks: An Introduction. Oxford University Press, Oxford.

Patel, R. R., and G. A. Riveros. 2013. Towards development of innovative bio-inspired materials by analyzing the hydrodynamic properties of polodon spathula (paddlefish) rostrum. ERDC/ITL TR-13-4. Vicksburg, MS: U.S. Army Engineer Research and Development Center.

Radjai, F., D. E. Wolf, M. Jean, and J. J. Moreau. 1998. Bimodal character of stress transmission in granular packings. Physical Review Letters 80(1):61-64.

Riveros, G. A., R. R. Patel, and J. J. Hoover. 2015. Swimming and Energy Dissipation Enhancement Induced by the Rostrum of the Paddlefish (Polyodon spathula): A Multiphysics, Fluid-Structure Interaction Analysis. Materials Research Society Fall Meeting.

Schrijver, A. 2002. On the history of the transportation and maximum flow problems. Mathematical Programming 91(3):437-445.

Segalman, D. J., C. W. G. Fulcher, G. M. Reese, and R. V. Field, Jr. 1998. An Efficient Method for Calculating RMS von Mises Stress in a Random Vibration Environment. Journal of Sound and Vibration 230(2):393-410. 
Tordesillas, A. 2007. Force chain buckling, unjamming transitions and shear banding in dense granular assemblies. Philosophical Magazine 87(32):4987-5016.

Tordesillas, A., D. M. Walker, E. Ando, and G. Viggiani. 2013. Revisiting localized deformation in sand with complex systems. Proc R Soc A 469: 20120606.

Tordesillas, A., D. M. Walker, G. Froyland, J. Zhang, and R. P. Behringer. 2012. Transition dynamics and magic-number-like behavior of frictional granular clusters. Physical Review E 86(1):011306.

Tordesillas, A., S. T. Tobin, M. Cil, K. Alshibli, and R. P. Behringer. 2015. Network flow model of force transmission in unbonded and bonded granular media. Physical Review E 91: 062204.

Vitali S., J. B. Glattfelder, and S. Battiston. 2011. The network of global corporate control. PLoS one 6(10):e25995. doi:10.1371/journal.pone.0025995.

Walker, D. M., and A. Tordesillas. 2010. Topological evolution in dense granular materials: a complex networks perspective. International Journal of Solids and Structures 47(5): 624-639. https://doi.org/10.1016/j.ijsolstr.2009.10.025. 


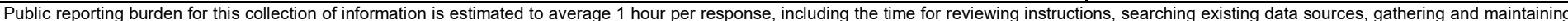

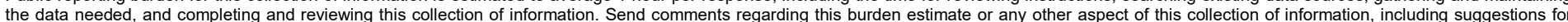

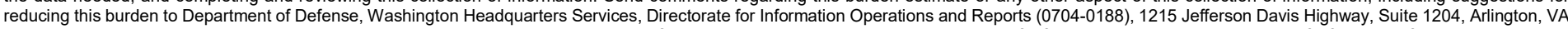

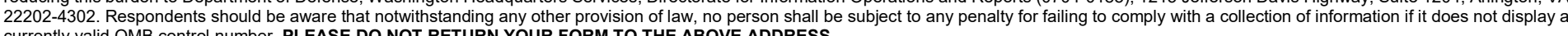
currently valid OMB control number. PLEASE DO NOT RETURN YOUR FORM TO THE ABOVE ADDRESS.
1. REPORT DATE (DD-MM-YYYY)
2. REPORT TYPE
October 2017
Final report

3. DATES COVERED (From - To)

\section{TITLE AND SUBTITLE}

Early Detection Of Failure Mechanisms In Resilient Biostructures: A Network Flow Study

5a. CONTRACT NUMBER

5b. GRANT NUMBER

5c. PROGRAM ELEMENT NUMBER

\section{AUTHOR(S)}

Reena R. Patel, Guillermo A. Riveros, Felipe J. Acosta, Edward J. Perkins, Jan J. Hoover, and David S. Thompson

5d. PROJECT NUMBER

WP \# 17-202

5e. TASK NUMBER

5f. WORK UNIT NUMBER

8. PERFORMING ORGANIZATION REPORT NUMBER

U.S. Army Engineer Research and Development Center, Information Technology

Laboratory 3909 Halls Ferry Road, Vicksburg, MS 39180-6199

ERDC TR-17-11

9. SPONSORING / MONITORING AGENCY NAME(S) AND ADDRESS(ES)

10. SPONSOR/MONITOR'S ACRONYM(S)

Headquarters, U.S. Army Corps of Engineers

Washington, DC 20314-1000

11. SPONSOR/MONITOR'S REPORT

NUMBER(S)

\section{DISTRIBUTION / AVAILABILITY STATEMENT}

Approved for public release; distribution unlimited.

\section{SUPPLEMENTARY NOTES}

\section{ABSTRACT}

The area of bioinspired material design is fairly young and mainly unexploited. This research aims to gain fundamental understanding of the hierarchical lattice architecture in the paddlefish rostrum and use this knowledge to obtain new insights for a wide range of applications. This work presents an integrated, interdisciplinary approach that employs computational mechanics and a complex network strategy to gain fundamental insights into the failure mechanisms of high performance, lightweight, structured composites by examining the geometry and material properties of the rostrum. Results from computational mechanics simulations and complex network analysis are presented with emphasis on the load transfer mechanism in the Bio-Structure, which is formation of force chains in structural system in the principal direction of the external stress to which the system is subjected. The current research presents a complex network analysis of the rostrum with and without the presence of the lattice architecture. Highly localized force chains were formed in the rostrum with similar loading conditions in absence of the lattice architecture. The flow network was also able to capture the stain localization in the tensile region of the rostrum and the asymmetrical response of the rostrum to uniform loading.

$\begin{array}{lll}\text { 15. SUBJECT TERMS } & \text { Biomechanics } & \text { Morphology } \\ & \text { Structural Analysis (Engineering) } & \text { Materials-Elastic properties } \\ & \text { Kinematics }\end{array}$

Stress concentration

16. SECURITY CLASSIFICATION OF:

a. REPORT

UNCLASSIFIED

b. ABSTRACT
UNCLASSIFIED

c. THIS PAGE

UNCLASSIFIED
17. LIMITATION OF ABSTRACT

UNCLASSIFIED

\section{NUMBER} OF PAGES

46 19a. NAME OF RESPONSIBLE PERSON R. R. Patel 19b. TELEPHONE NUMBER 6016345430 